\title{
The internal state of medium spiny neurons varies in response to different input signals
}

\author{
Zhen $\mathrm{Qi}^{1,2,3}$, Gary W Miller ${ }^{2,4}$, Eberhard O Voit ${ }^{1,3^{*}}$
}

\begin{abstract}
Background: Parkinson's disease, schizophrenia, Huntington's chorea and drug addiction are manifestations of malfunctioning neurons within the striatum region at the base of the human forebrain. A key component of these neurons is the protein DARPP-32, which receives and processes various types of dopamine and glutamate inputs and translates them into specific biochemical, cellular, physiological, and behavioral responses. DARPP-32's unique capacity of faithfully converting distinct neurotransmitter signals into appropriate responses is achieved through a complex phosphorylation-dephosphorylation system that evades intuition and predictability.

Results: To gain deeper insights into the functioning of the DARPP-32 signal transduction system, we developed a dynamic model that is robust and consistent with available clinical, pharmacological, and biological observations. Upon validation, the model was first used to explore how different input signal scenarios are processed by DARPP32 and translated into distinct static and dynamic responses. Secondly, a comprehensive perturbation analysis identified the specific role of each component on the system's signal transduction ability.

Conclusions: Our study investigated the effects of various patterns of neurotransmission on signal integration and interpretation by DARPP-32 and showed that the DARPP-32 system has the capability of discerning surprisingly many neurotransmission scenarios. We also screened out potential mechanisms underlying this capability of the DARPP-32 system. This type of insight deepens our understanding of neuronal signal transduction in normal medium spiny neurons, sheds light on neurological disorders associated with the striatum, and might aid the search for intervention targets in neurological diseases and drug addiction.
\end{abstract}

\section{Background}

The basal ganglia form a functional complex of neurons that are located at the base of the human forebrain. Malfunctioning of this complex has been associated with Parkinson's disease, schizophrenia, Huntington's chorea, and drug addiction [1]. The basal ganglia system receives most of its input through the striatum, which consists primarily of medium spiny neurons. These neurons are equipped with a protein, called DARPP-32 (dopamine- and cAMP-regulated phosphoprotein with $32 \mathrm{kDa}$ molecular weight), that is the key mediator of signal processing [2]. In response to dopamine signals, DARPP-32 mediates a variety of biochemical, cellular, and physiological effects $[3,4]$, including changes in the ion channel state of membranes, altered transcription factor activity, up- or down-regulation of gene

\footnotetext{
* Correspondence: eberhard.voit@bme.gatech.edu

'Department of Biomedical Engineering, Georgia Institute of Technology and Emory University Medical School, Atlanta, GA 30332, USA
}

expression, and reward [5,6]. Furthermore, DARPP-32 has important functions in behavior, such as changes in motor activity. In addition to dopamine signals, DARPP32 processes excitatory glutamatergic signals from the cortex, which are similarly crucial inputs to the striatum $[7,8]$. Thus, DARPP-32 serves as a centralized location for receiving, integrating and processing signals from several neurotransmitters and constitutes a key element in the important cortico-striato-pallido-thalamo-cortical loop.

Experimental evidence has shown that DARPP-32 functions by translating the incoming neuronal signals into distinct activity profiles of kinases and phosphatases. This translation process is based on phosphorylation and dephosphorylation at multiple sites of DARPP-32 and accomplished through a complicated signal transduction network that uses a second messenger system and a biochemical pathway activated by calcium (Figures 1 and 2). Specifically, when dopamine 


\section{Synaptic Cleft}

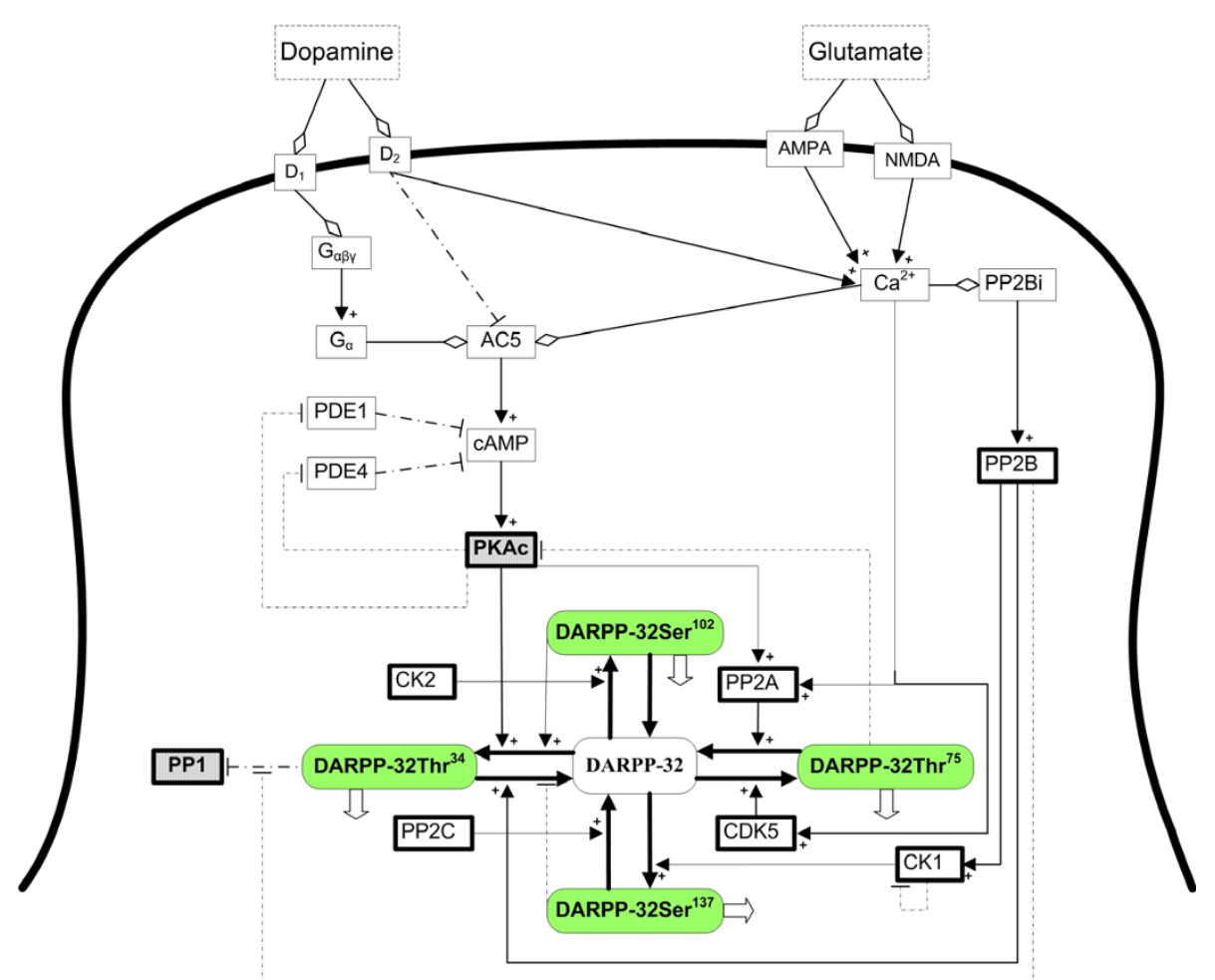

Figure 1 Signal integration and interpretation by the DARPP-32 system in dendrites of medium spiny neurons in the striatum. The membrane of spiny neurons (solid curved line) contains receptors for the neurotransmitters dopamine and glutamate, which had been released by other neurons into the synaptic cleft. Diamonds show molecular binding, while arrows with plus signs designate activation or enzymatic reactions and dash-dotted lines with bars indicate inhibition. Shaded boxes in green color represent phosphorylated forms of DARPP-32, with labels indicating the specific phosphorylated site, and arrows showing possible transitions between the different phosphorylation states. Open block arrows indicate possible multiple-site phosphorylation. Please refer to the Figure 2 for a detailed map of all possible transitions between different phosphorylation forms. Abbreviations are: dopamine receptor of $D_{1}$ subtype $\left(D_{1}\right)$, dopamine receptor of $D_{2}$ subtype $\left(D_{2}\right), G$ protein and subunits $\left(G_{\alpha \beta \gamma^{\prime}} G_{\alpha}\right)$, adenylate cyclase (AC5), cyclic AMP (CAMP), phosphodiesterases (PDE1, PDE4), protein kinase A (PKA, PKAc), alpha-amino-3hydroxy-5-methyl-4-isoxazolepropionic acid (AMPA), N-methyl-D-aspartate (NMDA), protein phosphatase 2B (PP2Bi, PP2B), protein phosphatase-1 (PP1), protein phosphatase 2A (PP2A), cyclin-dependent kinase 5 (CDK5), casein kinase 2 (CK2), casein kinase 1 (CK1), protein phosphatase $2 \mathrm{C}$ (PP2C), dopamine- and CAMP-regulated phosphoprotein with $32 \mathrm{kDa}$ molecular weight (DARPP-32). Phosphorylation of DARPP-32 is indicated by the corresponding amino acid and location, such as DARPP-32Thr ${ }^{34}$. See Figure 2 for multiple-site phosphorylation.

binds to its postsynaptic receptor of D1 subtype, a secondary messenger system is trigged that leads to the production of cyclic AMP (cAMP). This messenger molecule in turn activates protein kinase A (PKA). Both cAMP and PKA are critically regulated by phosphodiesterase (PDE), which has multiple isoforms such as PDE1 and PDE4 [9]. PKA then phosphorylates DARPP-32 at a specific threonine residue (Thr34) and thereby converts it into a potent inhibitor of protein phosphatase-1 (PP1) $[2,10]$. In contrast to dopamine, glutamate binds to its own ionotropic receptors (e.g., AMPA and NMDA), which causes $\mathrm{Ca}^{2+}$ cations to flow into the cell. This induced $\mathrm{Ca}^{2+}$ influx reduces the inhibition of $\mathrm{PP} 1$ by activating protein phosphatase $2 \mathrm{~B}$ (PP2B). PP2B dephosphorylates DARPP-32 at the threonine site and enhances the inhibition of PKA by increasing phosphorylation of DARPP-32 at another threonine residue, which is phosphorylated by cyclindependent kinase 5 (CDK5) [11-13].

The brief description of event chains indicates that the DARPP-32 signal transduction system balances the effects of competing triggers, which themselves are dynamic and depend on their recent histories. As a consequence, the integration and interpretation of neuronal signals by DARPP-32 can vary in response to different trains of input signals. In other words, different signal patterns, such as a single signal $v s$. coexistent signals, a one-time pulse $v s$. repetitive pulses, or signals for short-term $v s$. long-term plasticity, can have distinct effects on rewards, learning, memory, locomotion and/or synaptic plasticity.

The multitude of graded effects and interactions of the complex DARPP-32 system easily evades intuition and 


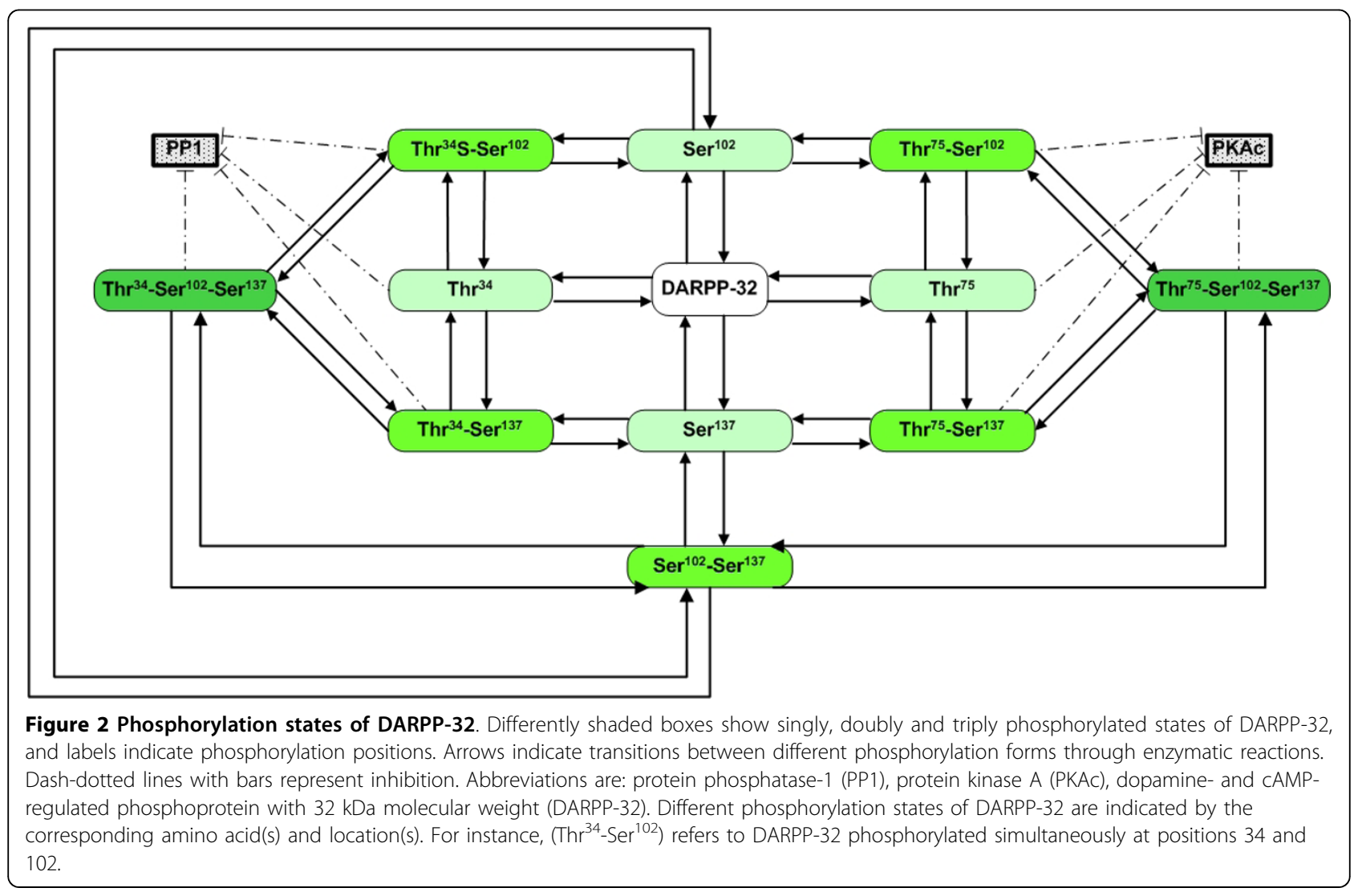

would benefit from a representation and analysis with methods of systems biology. Such an analysis could yield answers to questions regarding the specifics of the operation and design of the signal transduction system. For instance, one might ask which mechanisms are dominant in a given signaling situation and within a specific context. Do these mechanisms function synergistically or antagonistically? To what degree do they contribute to different modes of signal integration that are required by relevant brain functions or implicated in neurological diseases?

The goal of this work is to investigate such questions systematically and quantitatively with methods of computational systems biology. At the core of our approach is a dynamic model that is capable of accounting for the many components and processes associated with the DARPP-32 system in a numerical fashion. The details of this model are described in the Methods Section, and the model itself is presented explicitly and completely in Additional file 1. Upon formulating, testing, and validating the model, we simulated various patterns of neurotransmission and studied their effects on signal integration and interpretation by DARPP-32. The simulations and analyses addressed both the steady-state and the dynamics of the system. Moreover, systematic perturbations with the validated model revealed which mechanisms are dominant in specific signal input situations. This type of insight deepens our understanding of neuronal signal transduction in normal medium spiny neurons, could possibly shed light on mechanisms of neurological disorders associated with the striatum, and might aid the search for intervention targets in neurological diseases.

\section{Results}

\section{Validation of the model}

The model, as detailed in the Methods Section, serves as an exploratory platform for scenario simulations and their evaluations. However, before the model can be used in this capacity, it must be tested and validated against available experimental and clinical data on the biochemistry, pharmacology, and electrophysiology of the system. The available experimental observations cover both static and dynamic behaviors of the DARPP32 system. Most of them were carried out on rats.

Under normal, unstimulated base conditions, the degree of DARPP-32 phosphorylation at Thr34 and Thr75 was experimentally determined as $0.5-1.0 \%$ and $26 \%$, respectively [14-16]. Our model shows phosphorylation rates of $\sim 0.7 \%$ and $\sim 28.6 \%$ of total DARPP-32 molecules, respectively. The model also suggests that phosphorylation at Ser102 and Ser137, as well as 
unphosphorylated DARPP-32 account for $~ 3.0 \%$, $\sim 28.7 \%$, and $\sim 48.9 \%$, respectively. Meanwhile, active PKA and PP1 show levels of $\sim 0.7 \mathrm{nM}$ and $\sim 4.7 \mu \mathrm{M}$, respectively.

In laboratory experiments, the Thr34 phosphorylation level rose over 6 -fold within 2 to 10 minutes when the dopamine signal was activated following a stimulus [17-19], while Thr75 decreased to about half its base value [16]. Quite consistently, the model shows that Thr34 increases about 7 -fold within 3 minutes and Thr75 decreases by $\sim 35 \%$ following a 5 -minute stimulation of $10 \mu \mathrm{M}$ dopamine. In experiments where dopamine was depleted from the striatum, Thr75 (but not Thr34) rose substantially [20], and again this observation is reproduced well in a model simulation.

Similarly, the effects of stimulatory glutamate signals were assessed. Experiments indicated that an enhanced or prolonged glutamate signal increases the $\mathrm{Ca}^{2+}$ concentration, which in turn leads to a rapid $50 \%$ reduction in the levels of Thr34 and Thr75 [21,22]. In the corresponding model simulation of a 5-minute stimulation with $1 \mu \mathrm{M} \mathrm{Ca}^{2+}$, Thr34 and Thr75 phosphorylation is reduced to $\sim 30 \%$ and $\sim 55 \%$ of untreated levels within 1 minute. When the stimulus ceases, it takes about 6 minutes for Thr75 and 20 minutes for Thr34 to return to their basal levels $[21,22]$, and again these simulation results are consistent with experimental observations.

The dopamine and glutamate signals modulate each other's effects on DARPP-32. Using concurrent signals, the model shows that glutamate inhibits a Thr34 increase and enhances a Thr75 decrease induced by the dopamine signal, whereas dopamine counteracts a Thr34 decrease caused by glutamate and raises its level. Again, these simulation results are consistent with experimental findings [17].

Genetic and pharmacological interventions to the DARPP-32 system were also simulated with the model and the results were compared with experimental data. The effect of an intervention is simulated most readily by adjusting the numerical configuration of the model correspondingly. For example, $90 \%$ inhibition of PP2B by cyclosporin A is implemented as a $90 \%$ reduction in PP2B enzyme activity since the cyclosporin A is not in the model. Experiments showed that CDK5 inhibition by $10 \mu \mathrm{M}$ roscovitine reduced Thr75 by $\sim 73 \%$ and raised Thr34 about 10 fold [16,19,23,24]. Consistently, a model simulation of CDK5 inhibition showed that Thr75 decreases by $\sim 60 \%$, while Thr34 increases more than 7 fold.

Other experiments showed that CDK5 activation by up-regulation of its activator p35 attenuates the effects of cocaine-mediated dopamine transmission on raising Thr34 and reducing Thr75 [25]. This observation is also well reproduced by a model simulation. Furthermore, manipulations on some other important kinases and phosphatases are consistent with experimental observations. For instance, the model shows that $90 \%$ inhibition of PP2B by cyclosporin A eliminates the effect of $\mathrm{Ca}^{2+}$ on Thr34 phosphorylation [17], and that almost complete inhibition of PP2A by $1 \mu \mathrm{M}$ okadaic acid raises the Thr75 level by over 3 fold $[19,22,26]$.

\section{Effects of different signals}

After the model has been tested and proved to have the ability to reproduce diverse experimental observations, it can be utilized to study the characteristics and capabilities of the DARPP-32 system in integrating and interpreting different types of neuronal signals, as described in the Methods Section. The main goal of this analysis was to answer the question of how the DARPP-32 system manages to map different input scenarios reliably into distinct responses. In this section, we analyze the effects of different inputs on the state of the DARPP-32 system, while the following section will shed light on the internal mechanisms that implement this mapping. These modeling activities generate actual predictions since corresponding experiments have not yet been documented in the literature.

Relevant signals included dopamine and glutamate stimulation, and they were implemented by specifically resetting the extracellular dopamine level and the intracellular concentration of $\mathrm{Ca}^{2+}$, respectively. As discussed in detail in the Methods Section, we tested different input signal types, namely: individual or combined dopamine and glutamate signals; transient or sustained signals; one-shot pulse or repetitive pulse signals; signals of varying time scale; and signals of high or low amplitude. To configure these input signals quantitatively, we first assessed which time scales were most realistic. According to experimental measurements, the dopamine release stimulated by action potentials at different frequencies can have a time scale of between milliseconds $(<2 \mathrm{~Hz}$ stimuli) and seconds ( $\sim 20 \mathrm{~Hz}$ stimuli) [27]. The $\mathrm{Ca}^{2+}$ current responds to a glutamate signal as fast as milliseconds [28]. Accordingly, we set time scales of dopamine and calcium signals between milliseconds and seconds. In accordance with the frequencies of different brain waves, we set the input signals such that a sustained input has a 5-fold larger width than a transient input, and that a repetitive input comprises five pulses instead of one pulse in a one-shot input (Figure 3).

\section{Dopamine signals}

When the DARPP-32 system was stimulated with different patterns of dopamine signals, its responses were typically characterized by a spike in PKA and $\mathrm{D}_{34}$ * (DARPP-32 phosphorylated at Thr34), as well as a trough in PP1 and $\mathrm{D}_{75}$ * (DARPP-32 phosphorylated at Thr75) (Figure 4A). The amplitudes of these spikes and 


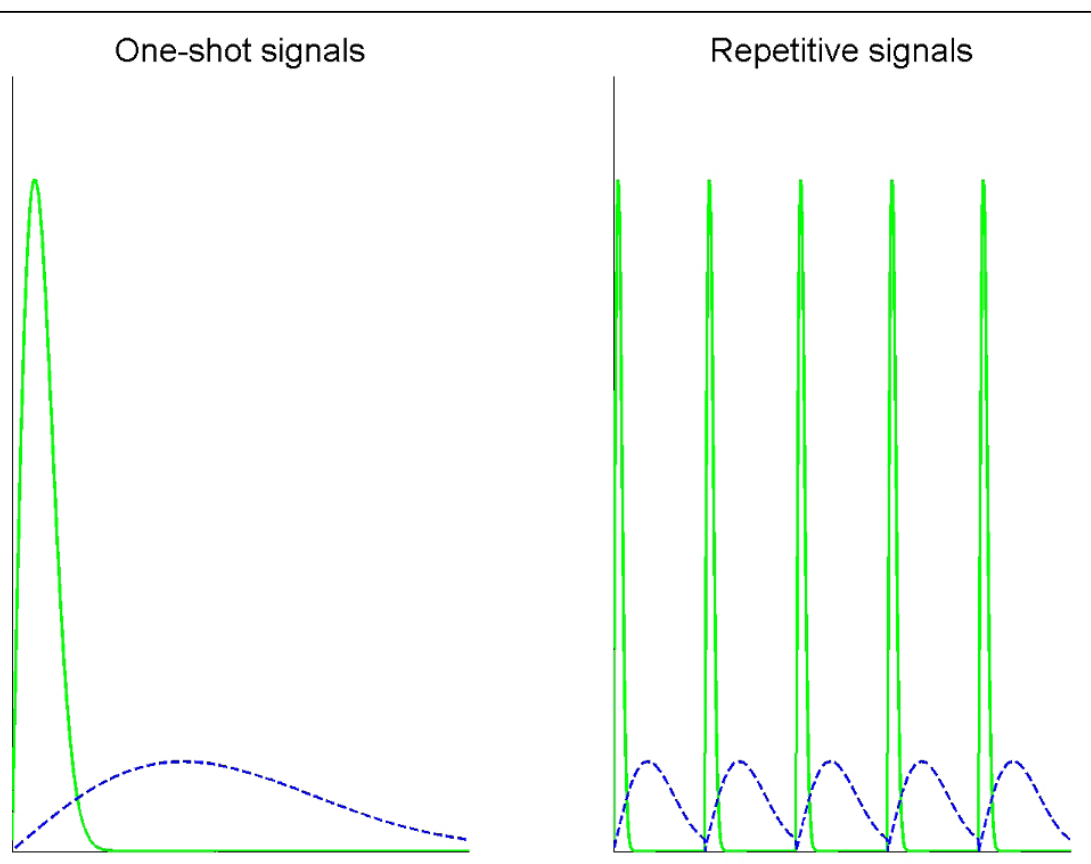

Figure 3 Schematic representation of input signal patterns. The left panel illustrates one-shot scenarios of input signals, while the right panel shows repetitive input signals. Solid lines represent transient signals, while dashed lines show sustained input signals.

troughs depended on duration, intensity, and shape of a signal.

\section{Glutamate signals}

Glutamate signals are represented in the model as changes in $\mathrm{Ca}^{2+}$ influx. In response to most types of $\mathrm{Ca}^{2+}$ influx, the DARPP-32 system commonly responded with spikes in both PKA and PP1, which were accompanied by troughs in $\mathrm{D}_{34}$ " and $\mathrm{D}_{75}$ * (Figure 4B). Compared to responses to dopamine signals, PKA exhibited an extra undershoot and consequently went through a refractory period, while $\mathrm{D}_{75}$ * showed an extra overshoot and a subsequent refractory period. The main distinctive feature between the effects of dopamine and those of glutamate is the qualitative difference in the dynamics of PP1 and $\mathrm{D}_{34}$ *.

\section{Combined signals}

When the system was exposed to simultaneous dopamine and glutamate signals, the DARPP-32 system responses often resembled the responses to $\mathrm{Ca}^{2+}$ signals alone (Figure 4C). However, the undershoot in PKA and its refractory period were no longer seen, and the overshoot in $\mathrm{D}_{75}$ * was less obvious or disappeared. The amplitude of the spike in PKA was determined mainly by the dopamine, while the amplitude of the trough in $\mathrm{D}_{75}$ * was mostly attributed to the $\mathrm{Ca}^{2+}$ flux.

\section{Types of signals}

Instead of inducing typical responses as described above, some signal patterns qualitatively altered the steady state of the DARPP-32 system or caused unusual dynamic behaviors. To code the characteristics of a signal pattern in a succinct notation, we use in the following the notation [signal time scale (milliseconds or seconds)/signal number (one-shot or repetitive)/signal shape (transient or sustained)/signal amplitude (high or low amplitude)].

\section{Transient vs. sustained}

A transient $\mathrm{Ca}^{2+}$ signal [milliseconds/repetitive/transient/high amplitude] had the capability of switching the DARPP-32 system to a new steady state, which was characterized by elevated PP1 and reduced levels of PKA, $D_{34}$ *, and $D_{75}$ *. This switch should be seen in contrast to the complete recovery of the steady state when the corresponding sustained input signal was applied (Figure 5).

\section{One-shot vs. repetitive}

A one-shot pulse can induce distinctly different responses of the DARPP-32 system than the corresponding repetitive signal. Figure 6 shows such an example in the case of a combined signal of dopamine and $\mathrm{Ca}^{2+}$ : A single pulse (dopamine combined with $\mathrm{Ca}^{2+}$, seconds/one-shot/sustained/low amplitude) caused a trough in PP1, which is in stark contrast to a spike if the input was a repetitive signal. Also, the one-shot signal induced a spike in $\mathrm{D}_{34}{ }^{*}$, while the repetitive pulse caused a trough. In addition, the spike in $D_{75}$ * was almost erased in the one-shot scenario.

High vs. low amplitude

A change in signal amplitude can qualitatively alter the dynamic responses of the DARPP-32 system, as it is 

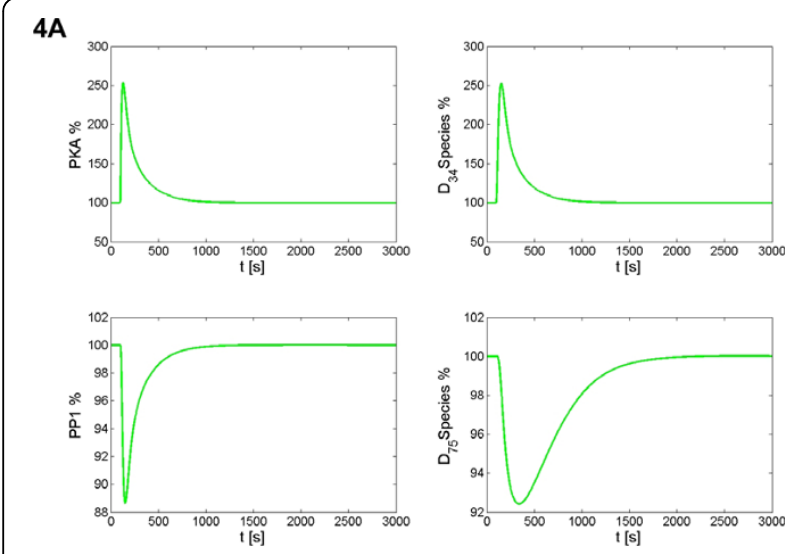

4B
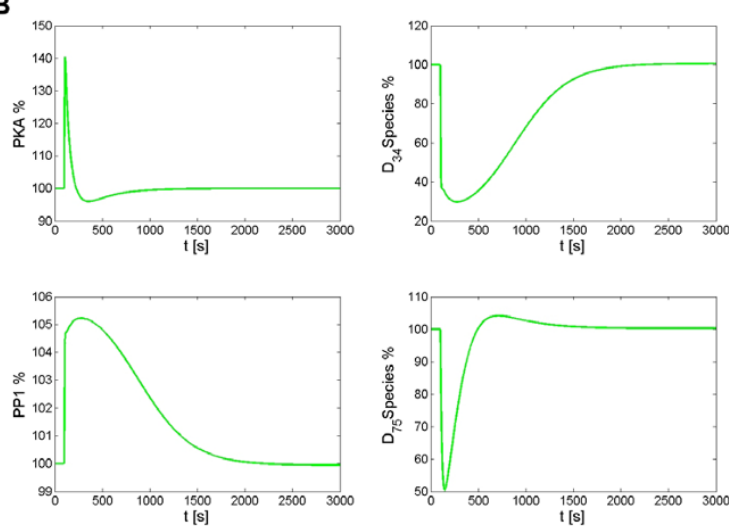

$4 \mathrm{C}$
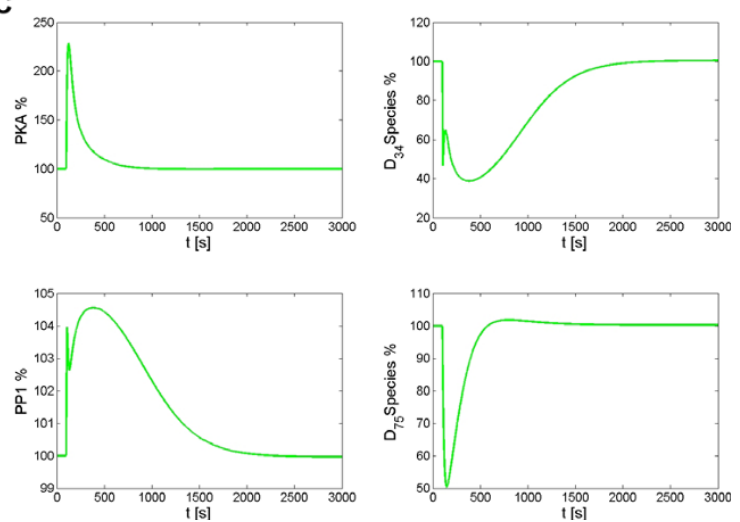

Figure 4 Typical responses of the DARPP-32 system to dopamine and glutamate $\left(\mathrm{Ca}^{2+}\right.$ influx) signals. Panel A shows a typical response of the system to a sole dopamine signal, while panel $B$ exhibits a representative response of the system to a sole glutamate $\left(\mathrm{Ca}^{2+}\right.$ influx $)$ signal. Panel $\mathrm{C}$ shows a common response of the system to a combination of dopamine and $\mathrm{Ca}^{2+}$ signals. shown in Figure 7. A $\mathrm{Ca}^{2+}$ signal with high amplitude (seconds/repetitive/sustained/high amplitude) induced a spike and a refractory period in PKA, while the corresponding signal of low amplitude had almost no effect on PKA. The high amplitude signal caused a trough in $\mathrm{D}_{75}{ }^{*}$, while the low amplitude signal had the opposite effect. An input signal of high amplitude could also have a different effect on the steady state of the DARPP-32 system than the corresponding signal of low amplitude. Figure 8 shows that a combined signal of dopamine and $\mathrm{Ca}^{2+}$ (milliseconds/repetitive/transient/high amplitude) alters the steady state of the DARPP-32 system while the counterpart signal of low amplitude does not. The new steady state shown in the high amplitude scenario has an elevated level of PP1 and reduced level of PKA, $\mathrm{D}_{34}{ }^{*}$, and $\mathrm{D}_{75}{ }^{*}$. The time scale is also important; however, its effects could be represented by variations in amplitude and the number of pulses of a signal.

Summarizing this section we can state that different patterns of input signals are clearly differentiated by the DARPP-32 signal transduction system and that they are reflected in the response state of the system ( $c f$. Table $1)$. The next section investigates the robustness of the stimulus-response pattern implementation against perturbations of its internal mechanisms.

\section{Robustness of signal-response implementation in the DARPP-32 system}

The qualitative differences seen in the static or dynamic behaviors of the DARPP-32 system in response to different signal patterns raise an interesting question, namely: What are the governing mechanisms responsible for these input-output differences and how robust are they? It is clear that the distinct system responses are critical for the proper functioning of the DARPP-32 signaling network, and that a better elucidation of their mechanistic implementation and robustness could possibly help deepen our understanding of its functioning, identify weak points in the system that are associated with disease outcomes, and provide targets for therapeutic interventions. Thus, in this section we employ systematic perturbations to analyze mechanisms that connect specific signals with the corresponding responses.

In order to assess the robustness of DARPP-32 mediated signal transduction, we simulated perturbations of all processes within the DARPP-32 system one by one to discover the most critical processes. We 


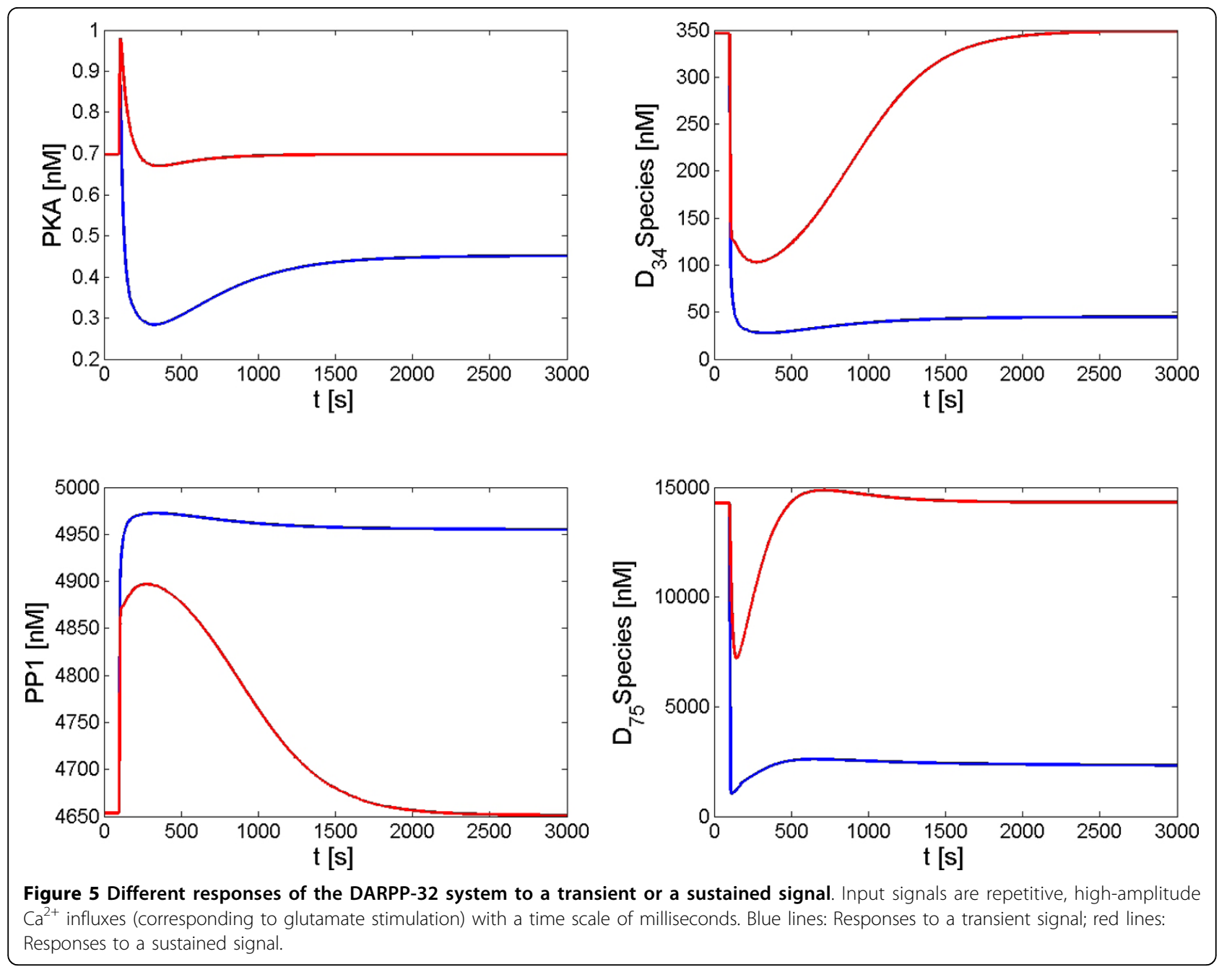

used signal configurations shown in Figures 5, 6, 7, and 8 as signal scenarios and in the following describe their implementation under the categories signal shape scenario, signal number scenario, signal amplitude scenario - dynamics, and signal amplitude scenario steady state. For each scenario, each process within the DARPP-32 system was separately accelerated or decelerated (including complete blockage) to simulate effects of chemical or genetic perturbations. The effect of each perturbation was assessed, thereby revealing the dominant processes of the signal-response implementation in each scenario.

\section{Signal shape scenario (transient vs. sustained)}

The most important result of this line of simulations affirms the extraordinary robustness of the DARPP-32 system. We systematically altered every process within the system within a range of 10 -fold acceleration or deceleration and found that no single process was so critical that such an alteration in its flux would eliminate the system's ability to discern between a transient and a sustained $\mathrm{Ca}^{2+}$ signal; the appropriate steady states of PKA and PP1 were achieved in all cases. Even under complete inhibition of a process, the DARPP-32 system usually achieved a different steady state when it was subjected to different $\mathrm{Ca}^{2+}$ signals.

However, there are crucial processes that can destroy the proper response pattern when sufficiently inhibited. Not surprisingly, processes transducing the $\mathrm{Ca}^{2+}$ signal are very important. Especially, blockade of activation of PP2B by the $\mathrm{Ca}^{2+}$ signal can eliminate the differences in steady states and thereby prevent the system from distinguishing these signals and responding appropriately. Although these inputs are associated with $\mathrm{Ca}^{2+}$ signals, which correspond to glutamatergic stimulation, the dopamine signal can have a crucial function in this context. Specifically, inhibition of processes for cAMP and PKA production can equalize responses induced by transient and sustained $\mathrm{Ca}^{2+}$ signals and thereby compromise signal discernibility. Because of the important roles of the dopamine pathway and PKA, the inhibition 


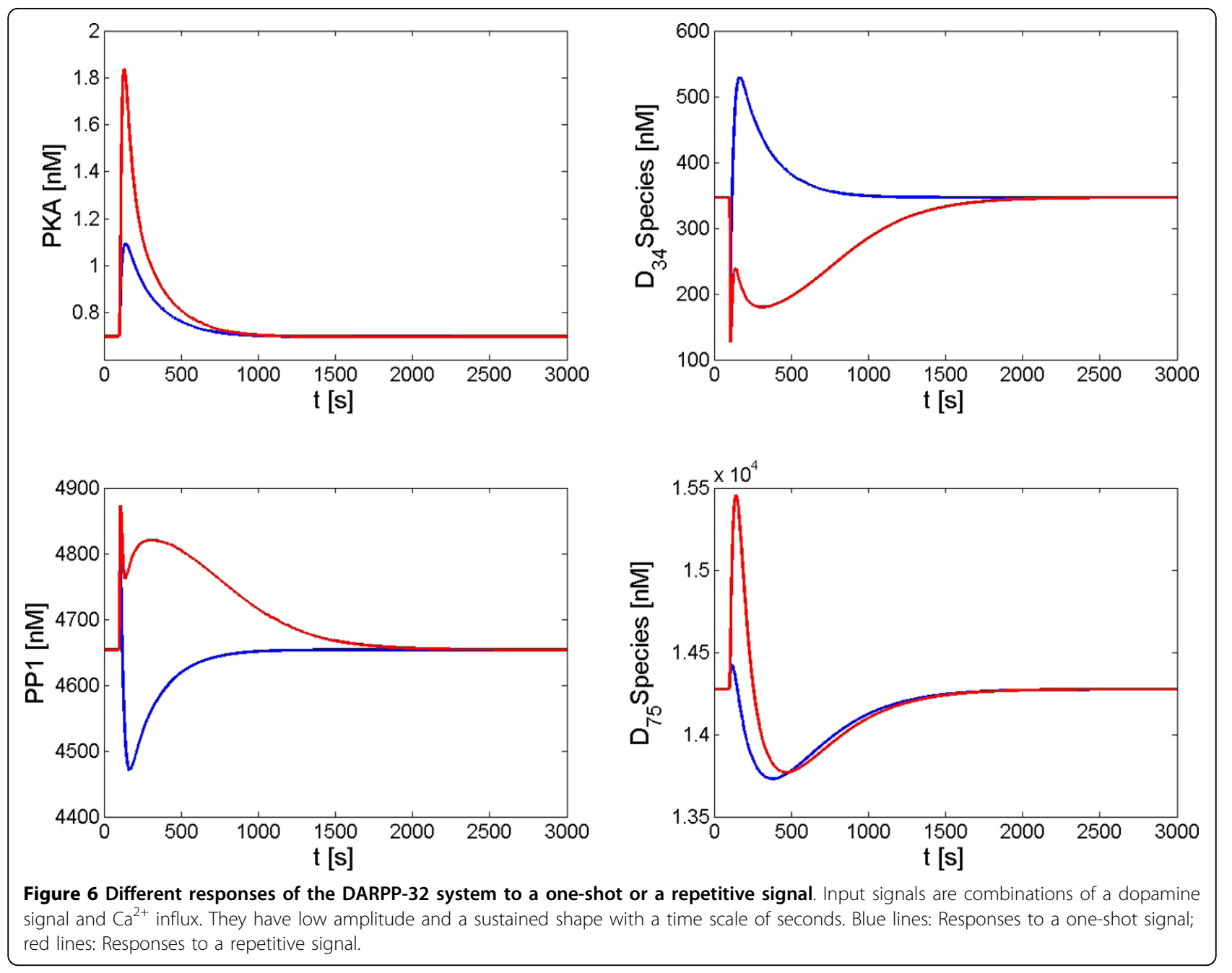

of PKA by $\mathrm{D}_{75}$ phosphorylation also contributes substantially to variations in the steady state of the DARPP32 system.

\section{Signal number scenario (one-shot vs. repetitive signal)}

Simulations screening for critical processes in this category suggest that the dopamine pathway is crucial for dynamic differences, especially the production processes of cAMP and PKA. Phosphodiesterases, which inhibit the formation of cAMP, similarly make an important contribution to these differences. The $\mathrm{Ca}^{2+}$ pathway, and specifically its activation of $\mathrm{PP} 2 \mathrm{~B}$, is also critical for discerning a one-shot from a repetitive signal.

\section{Signal amplitude scenario (high vs. low amplitude)}

Finally, the critical processes for mediating different signal amplitudes and transducing them to distinct dynamic transients and steady states of the DARPP-32 system were investigated. The dopamine pathway and $\mathrm{Ca}^{2+}$ were both found to be significant contributors. However, the activation of PP2B was no longer identified as crucial. Instead, $\mathrm{Ca}^{2+}$ activation of the phosphatase PP2A and the interaction between PKA and PP2A are dominant for both dynamic and static responses to different signal amplitudes. Although phosphodiesterases can inhibit the formation of cAMP and thus reduce the activity of PKA, they contribute only negligibly to the observed differences in response to high and low signal amplitudes.

\section{Discussion}

The DARPP-32 system integrates different types of dopamine and glutamate signals and transduces them toward appropriate biochemical, cellular, physiological, and behavioral responses, such as motor behavior [29]. It mediates these crucial functions of neurotransmitters through regulating the phosphorylation profiles of PKA and PP1 in an appropriate and adaptive fashion [3,30]. For instance, by means of phosphorylation or dephosphorylation of the glutamate receptors, an incoming neuronal signal can induce changes in the conductance and/or density of these receptors, thereby changing the 


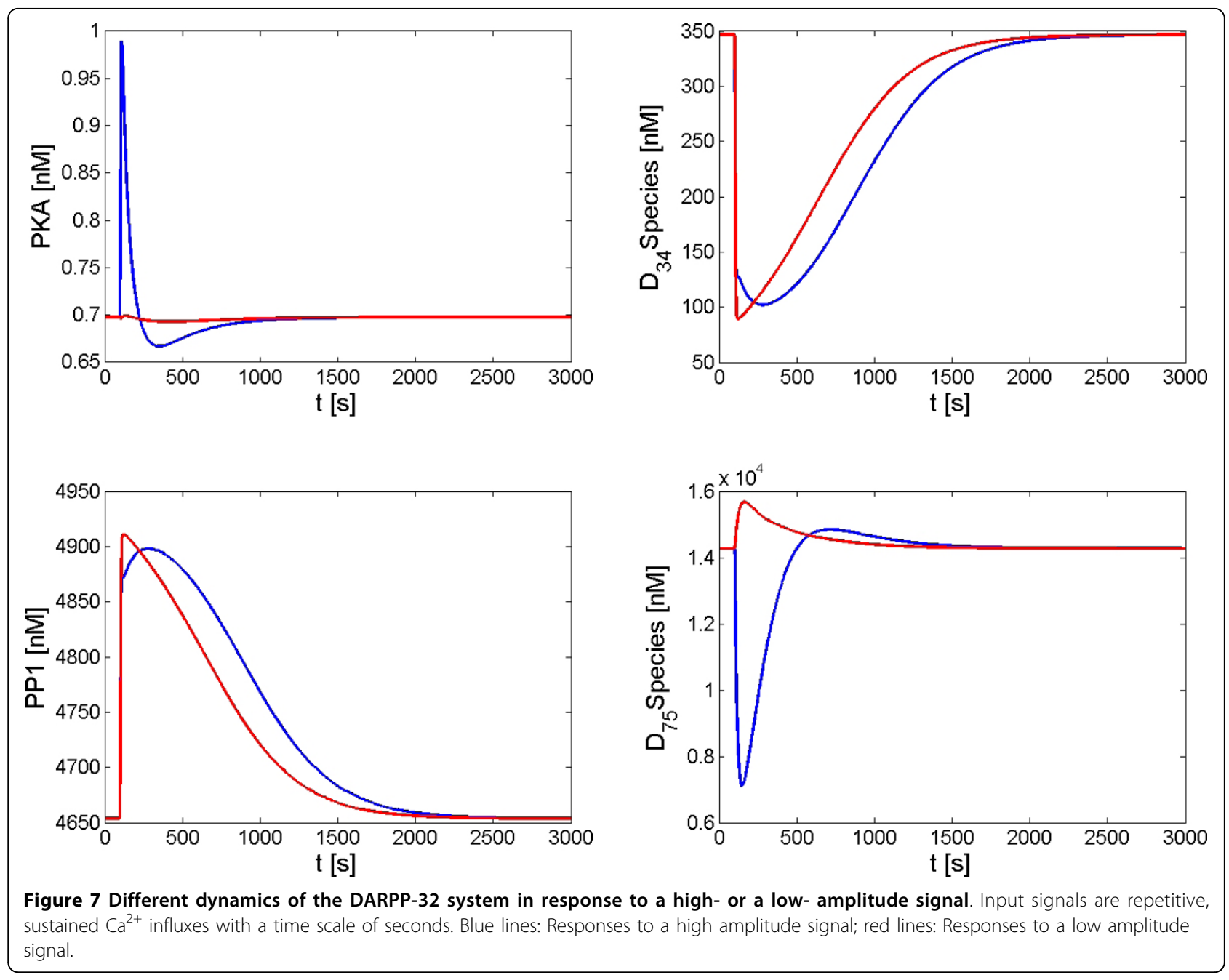

cell's responsiveness adaptively [31-33]. Also, N/P-type $\mathrm{Ca}^{2+}$ channels and neuronal $\mathrm{Na}^{+}, \mathrm{K}^{+}$-ATPase can be regulated by neurotransmitters via the DARPP-32 system [5]. Furthermore, experiments of DARPP-32 knockout mice demonstrated that DARPP-32 contributes to synaptic plasticity such as long-term depression and long-term potentiation [34]. These long-term forms of synaptic plasticity can be induced by altering gene transcription for synaptic function through PKA and PP1 regulation of transcription factors such as CREB and of immediate early genes such as $\mathrm{C}$-fos and $\Delta$ fosB [5,35-37]. In addition, locomotor activity and reward behavior can be regulated through the DARPP-32 system.

Thus, a vast array of studies has confirmed that the DARPP-32 system is a critical mediator for complex neuronal signal transduction. Combining this documented function with our detailed model analyses showing how various patterns of neurotransmission have distinct effects on the internal state of the DARPP-32 system raises an important question, namely: Are the different biochemical states and transient responses of DARPP-32 physiologically and behaviorally significant and could they even correspond to different phenotypes associated with striatal function and dysfunction? Complete answers cannot yet be given and will require further exploration and discovery through cell and animal experiments. A biological phenomenon of relevance in this category is reinforcement learning, with which medium spiny neurons in the striatum can fire more easily under sole cortical input. This functionality is achieved through phosphorylation of AMPA receptors, which can be phosphorylated and dephosphorylated at Ser845 of its GluR1 subunit by PKA and PP1, respectively. This type of phosphorylation and dephosphorylation activity corresponds to the insertion and removal of membrane associated AMPA receptors, respectively [38,39]. Since our model suggests that different input signals can lead to distinct PKA and PP1 activity profiles, it might be 


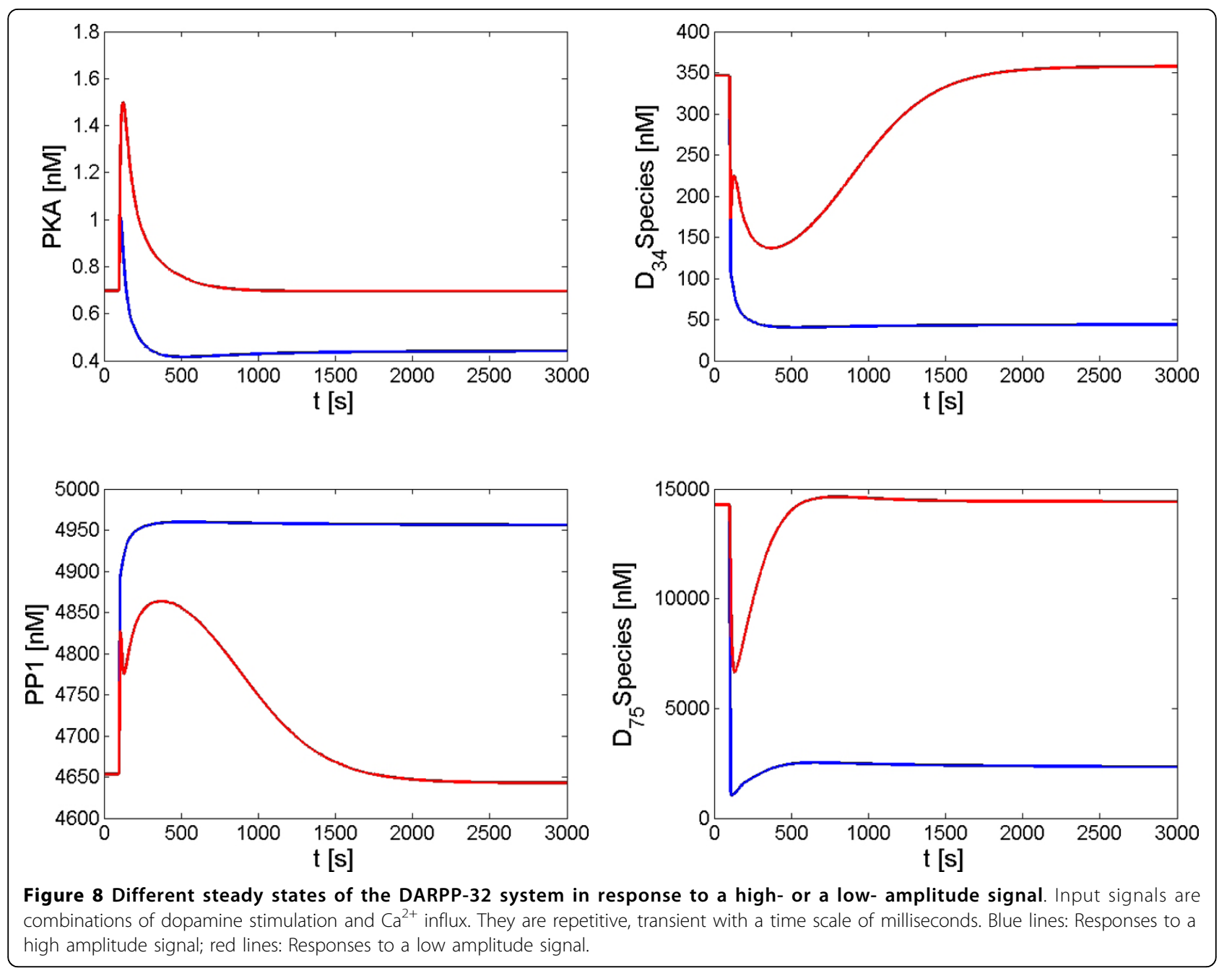

worthwhile exploring the connections of these simulated signals with reinforcement learning.

Systematic simulations of local perturbations affecting the DARPP-32 system demonstrated that the system is very robust in its interpretation of the relevant spectrum of neuronal signals. Even under conditions of 10-fold acceleration or deceleration of any single process, the DARPP-32 system was still able to translate input signals into their correct responses. However, the system also contains critical components whose perturbation could interrupt normal behaviors. Among these are mechanisms transducing the $\mathrm{Ca}^{2+}$ signal, especially the activation of PP2B. If this activation is impeded, the dynamics and/or static states of the system may no longer reflect the correct input signal.

The model demonstrates in what manner the dopamine pathway plays an indispensable role in signal interpretation. The primary mechanism is the regulation of the kinase PKA through the production of cAMP and the phosphorylation level of DARPP-32 at the Thr75 site. Depending on input signal patterns, dopamine and glutamate can have synergistic or antagonistic interactions, which contribute to distinctly different responses.

The work presented here focuses primarily on the neurotransmitters dopamine and glutamate. For medium spiny neurons which receive innervation from excitatory glutamatergic neurons and modulatory dopaminergic neurons, our results showed that these two neurotransmitters interact differently within the DARPP-32 system in response to various input signal patterns. Meanwhile, different transmitter systems contribute most to observed discerning capability of different signal patterns. Thus, if an intervention is designed to manipulate signal discernibility by the DARPP-32 system, the choice of the targeted neurotransmitter system has to be based on the specific goal. For such a task, our model can be used to screen the target and simulate the effects of the intentional intervention.

It is known that medium spiny neurons also receive inputs from other neurotransmitters such as serotonin, 
Table 1 Mapping capability of the DARPP-32 system between neuronal signals and phosphorylation profiles of PKA and PP1

\begin{tabular}{|c|c|c|c|c|c|}
\hline \multicolumn{2}{|c|}{ Input Signal } & \multicolumn{2}{|c|}{$\begin{array}{l}\text { DARPP-32 } \\
\text { Status }\end{array}$} & \multicolumn{2}{|c|}{ Output } \\
\hline Dopamine $^{a}$ & Glutamate $^{a}$ & $\mathrm{Thr}_{34}{ }^{\mathrm{b}}$ & $\mathrm{Thr}_{75}{ }^{\mathrm{b}} 75$ & $\mathrm{PKA}^{\mathrm{b}}$ & $\mathrm{PP}^{\mathrm{b}}$ \\
\hline \multirow{15}{*}{$\begin{array}{l}\text { Any signal of } \\
\text { millisecond to second } \\
\text { scale }\end{array}$} & & $\Delta \equiv$ & $\nabla \equiv$ & $\Delta \equiv$ & $\nabla \equiv$ \\
\hline & ms-sp-tt-ha & $\nabla \downarrow$ & $\nabla \downarrow$ & $\nabla \downarrow$ & $\Delta \uparrow$ \\
\hline & ms-sp-tt-la & $\nabla \equiv$ & $\nabla \equiv$ & $\Delta \equiv$ & $\Delta \equiv$ \\
\hline & ms-sp-st-ha & $\nabla \equiv$ & $\nabla \equiv$ & $\Delta \equiv$ & $\Delta \equiv$ \\
\hline & ms-sp-st-la & $\nabla \equiv$ & $\nabla \equiv$ & $\leftrightarrow \equiv$ & $\Delta \equiv$ \\
\hline & ms-rp-tt-ha & $\nabla \downarrow$ & $\nabla \downarrow$ & $\nabla \downarrow$ & $\Delta \uparrow$ \\
\hline & ms-rp-tt-la & $\nabla \equiv$ & $\nabla \equiv$ & $\Delta \equiv$ & $\Delta \equiv$ \\
\hline & ms-rp-st-ha/la & $\nabla \equiv$ & $\nabla \equiv$ & $\Delta \equiv$ & $\Delta \equiv$ \\
\hline & s-sp-tt-ha & $\nabla \equiv$ & $\nabla \equiv$ & $\Delta \equiv$ & $\Delta \equiv$ \\
\hline & s-sp-tt-la & $\nabla \equiv$ & $\nabla \equiv$ & $\leftrightarrow \equiv$ & $\leftrightarrow \equiv$ \\
\hline & s-sp-st-ha & $\nabla \equiv$ & $\nabla \equiv$ & $\Delta \equiv$ & $\Delta \equiv$ \\
\hline & s-sp-st-la & $\nabla \equiv$ & $\leftrightarrow \equiv$ & $\leftrightarrow \equiv$ & $\Delta \equiv$ \\
\hline & s-rp-tt-ha/la & $\nabla \equiv$ & $\nabla \equiv$ & $\Delta \equiv$ & $\Delta \equiv$ \\
\hline & s-rp-st-ha & $\nabla \equiv$ & $\nabla \equiv$ & $\Delta \equiv$ & $\Delta \equiv$ \\
\hline & s-rp-st-la & $\nabla \equiv$ & $\Delta \equiv$ & $\leftrightarrow \equiv$ & $\Delta \equiv$ \\
\hline ms-sp-tt-ha & ms-sp-tt-ha & $\nabla \downarrow$ & $\nabla \downarrow$ & $\nabla \downarrow$ & $\Delta \uparrow$ \\
\hline ms-sp-tt-la & ms-sp-tt-la & $\nabla \equiv$ & $\nabla \equiv$ & $\Delta \equiv$ & $\Delta \equiv$ \\
\hline ms-sp-st-ha/la & ms-sp-st-ha/la & $\nabla \equiv$ & $\nabla \equiv$ & $\Delta \equiv$ & $\Delta \equiv$ \\
\hline ms-rp-tt-ha & ms-rp-tt-ha & $\nabla \downarrow$ & $\nabla \downarrow$ & $\nabla \downarrow$ & $\Delta \uparrow$ \\
\hline ms-rp-tt-la & ms-rp-tt-la & $\nabla \equiv$ & $\nabla \equiv$ & $\Delta \equiv$ & $\Delta \equiv$ \\
\hline ms-rp-st-ha/la & ms-rp-st-ha/la & $\nabla \equiv$ & $\nabla \equiv$ & $\Delta \equiv$ & $\Delta \equiv$ \\
\hline s-sp-tt-ha & s-sp-tt-ha & $\nabla \equiv$ & $\nabla \equiv$ & $\Delta \equiv$ & $\Delta \equiv$ \\
\hline s-sp-tt-la & s-sp-tt-la & $\Delta \equiv$ & $\nabla \equiv$ & $\Delta \equiv$ & $\nabla \equiv$ \\
\hline s-sp-st-ha/la & s-sp-st-ha/la & $\Delta \equiv$ & $\nabla \equiv$ & $\Delta \equiv$ & $\nabla \equiv$ \\
\hline s-rp-tt-ha/la & s-rp-tt-ha/la & $\nabla \equiv$ & $\nabla \equiv$ & $\Delta \equiv$ & $\Delta \equiv$ \\
\hline s-rp-st-ha/la & s-rp-st-ha/la & $\nabla \equiv$ & $\nabla \equiv$ & $\Delta \equiv$ & $\Delta \equiv$ \\
\hline
\end{tabular}

${ }^{a}$ : Abbreviations for neurotransmission patterns are: millisecond (ms), second $(\mathrm{s})$, a single pulse (sp), repetitive pulses (rp), transient transmission (tt), sustained transmission (st), high amplitude signal (ha), and low amplitude signal (la).

$b$ : The responses of the DARPP-32 state and its output are presented as transient dynamics and long-term state. The transient dynamics is described as trough $(\nabla)$, essentially flat $(\leftrightarrow$, and peak $(\Delta)$ reflecting the main characteristic of the response. The long-term state of phosphorylation is given in relation to the resting level as decreased $(\downarrow)$, basal $(\equiv)$, and increased $(\uparrow)$.

and it is reasonable to expect that these signals also contribute significantly to functions of medium spiny neurons. These contributions will be the subject of future research. It might also be useful to expand the signal patterns proposed here. For instance, long-term behaviors lasting minutes, hours, or even longer are clearly biologically significant and should be studied with the proposed model as well. Finally, all neurotransmitter signals are controlled by action potentials, ion fluxes, and metabolic pathways in presynapses, where the neurotransmitters are synthesized, degraded, compartmentalized, released, and taken back into a synaptic terminal [40]. Thus, presynapse and postsynapse models should be linked in order to account for a more comprehensive understanding of normal function and dysfunction of the striatum.

\section{Conclusions}

In this study, we investigated systematically and quantitatively the capability of a signal transduction system (the DARPP-32 system) in neuronal signals integration and interpretation with methods of computational systems biology. As shown by our results, the DARPP-32 system implements a sophisticated many-to-many mapping that senses various signal characteristics and their combinations in terms of their biochemical source, shape, amplitude, repetition, and time scale and converts these into distinct internal states. These states correspond initially to distinct phosphorylation profiles of the DARPP-32 molecule and are subsequently translated into different dynamic and static activity configurations of the kinase PKA and the phosphatase PP1.

The amplitude of a signal is a direct function of the intensity of neurotransmitter release and contributes to synaptic plasticity. According to our results, different dopamine and glutamate signal amplitudes can cause the DARPP-32 to switch among its different steady states and/or to change its dynamic responses qualitatively. Similarly, neurotransmissions of different shapes and repetition characteristics can be translated through DARPP-32 into different steady states and/or distinct dynamic responses. Such dicernibility might have important implications in physiological and behavioral phenomena, such as synaptic plasticity.

The discernibility of the DARPP-32 system between various neurotransmission patterns is associated by our systemic analyses with mechanisms transducing the $\mathrm{Ca}^{2+}$ signal, especially the activation of PP2B, and mechanisms regulating the kinase PKA through the production of cAMP and the phosphorylation level of DARPP-32 at the Thr75 site.

These mechanisms potentially underlying the discernibility of the DARPP-32 system need to be verified experimentally. If the experiments confirm our predictions, these dominant processes have implications for the vulnerability of the system and for possible therapeutic interventions in Parkinson's disease, schizophrenia, and drug addiction. For example, the DARPP-32 system is involved in the generation of dyskinesia, a severe disorder often associated with Parkinson's disease [41]. It will be of great potential impact to test whether interventions targeting these critical mechanisms in the DARPP-32 system can ameliorate this disorder. Efforts are currently in progress to develop drugs acting on medium spiny neurons [42-44], and it 
appears that our model and simulation results could facilitate a deeper and more detailed understanding of the specific effects of these drugs. By simulating the drug mechanism, such model analyses could investigate not only the effects on the anticipated target but also on all other processes that are affected within the DARPP-32 system. In addition, one could study with relative ease how such effects of the drug vary in different context of neuronal signals. Upon further testing and refinements, which will require additional data and information, the model will have the potential of assessing the effects of drug interventions on targets and processes located downstream of the DARPP-32 system.

\section{Methods}

The DARPP-32 system includes various types of interactions, such as ligand-receptor binding, enzymatic reactions, activation, and inhibition. Our model, which accounts for all these components, is based on models developed by Lindskog and collaborators, the LeNovère's lab, and the Greengard's group [45-47], who studied various static and dynamic behaviors of the DARPP-32 system, but combines and extends these in a significant fashion. Its connectivity diagram is shown in Figures 1 and 2, and all mass action reactions are listed in Tables 2 and 3. A full, explicit and directly downloadable description of the model is available in Additional file 1.

Table 2 Reactions and rate constants of signal transduction for DARPP-32 phosphorylation in dendrites of medium spiny neurons in the striatum (see legend of Figure 1 for abbreviations)

\begin{tabular}{|c|c|c|c|c|}
\hline Reaction & $\mathrm{K}_{\mathrm{f}}\left(\mathrm{nM}^{-1} \cdot \mathrm{s}^{-1}\right)^{\#}$ & $K_{b}\left(s^{-1}\right)$ & $\mathrm{K}_{\mathrm{c}}\left(\mathrm{s}^{-1}\right)$ & Ref. \\
\hline $\mathrm{D} 1+\mathrm{DA} \leftrightarrow \mathrm{D} 1 \_\mathrm{DA}$ & $1.1 \mathrm{E}-3$ & 10.0 & & {$[47]$} \\
\hline$D 1 \_D A+G_{\alpha \beta \gamma} \leftrightarrow$ D1_DA_G $G_{\alpha \beta \gamma}$ & $6.0 \mathrm{E}-4$ & $1.0 \mathrm{E}-3$ & & [47] \\
\hline $\mathrm{D} 1+\mathrm{G}_{\alpha \beta \gamma} \leftrightarrow \mathrm{D} 1 \mathrm{G}_{\alpha \beta \gamma}$ & $6.0 \mathrm{E}-5$ & $3.0 \mathrm{E}-4$ & & [47] \\
\hline$D 1 \_G_{\alpha \beta \gamma}+D A \leftrightarrow D 1 \_D A \_G_{\alpha \beta \gamma}$ & $3.3 \mathrm{E}-3$ & 10.0 & & [47] \\
\hline D1_DA_G $G_{\alpha \beta \gamma} \rightarrow$ D1_DA $+G_{\alpha} G T P+G_{\beta \gamma}$ & $20.0^{\mathrm{a}}$ & & & [47] \\
\hline $\mathrm{G}_{\alpha} \mathrm{GTP} \rightarrow \mathrm{G}_{\alpha} \mathrm{GDP}$ & $10.0^{\mathrm{a}}$ & & & [47] \\
\hline $\mathrm{G}_{\alpha} \mathrm{GDP}+\mathrm{G}_{\beta \gamma} \rightarrow \mathrm{G}_{\alpha \beta \gamma}$ & 100.0 & & & [47] \\
\hline$G_{\alpha} G T P+A C 5 \leftrightarrow G_{\alpha} G T P \_A C 5$ & $3.9 \mathrm{E}-2$ & 50.0 & & [47] \\
\hline$G_{\alpha} G T P \_A C 5+$ ATP $\leftrightarrow G_{\alpha} G T P \_A C 5$ _ATP & $1.3 \mathrm{E}-4$ & $2.6 \mathrm{E}-1$ & & [47] \\
\hline$G_{\alpha} G T P \_A C 5$ _ATP $\leftrightarrow G_{\alpha} G T P \_A C 5+$ CAMP & $28.5^{\mathrm{a}}$ & $2.6 \mathrm{E}-4^{\mathrm{b}}$ & & [47] \\
\hline PKA +2 CAMP $\leftrightarrow$ PKA_CAMP 2 & $1.3 \mathrm{E}-5^{\mathrm{c}}$ & $6.0 \mathrm{E}-3$ & & [47] \\
\hline PKA_CAMP $_{2}+2$ CAMP $\leftrightarrow$ PKA_CAMP $_{4}$ & $1.7 \mathrm{E}-5^{\mathrm{c}}$ & $6.0 \mathrm{E}-2$ & & [47] \\
\hline $\mathrm{PKA}_{-} \mathrm{CAMP}{ }_{4} \leftrightarrow 2 \mathrm{PKAC}+\mathrm{PKAr}$ & $5.1 \mathrm{E}-4^{\mathrm{a}}$ & $4.8 \mathrm{E}-3^{\mathrm{c}}$ & & [47] \\
\hline PDE1 + CAMP $\leftrightarrow$ PDE1_CAMP $\rightarrow$ PDE1 + AMP & $2.0 \mathrm{E}-2$ & 72.0 & 18.0 & [47] \\
\hline PDE4 + CAMP $\leftrightarrow$ PDE4_CAMP $\rightarrow$ PDE4 + AMP & $2.0 \mathrm{E}-2$ & 72.0 & 18.0 & [47] \\
\hline $\mathrm{PKAc}+\mathrm{PDE} 1 \leftrightarrow \mathrm{PKAc}$ PPE $1 \rightarrow \mathrm{PKAc}+\mathrm{PDE} 1 \mathrm{p}$ & $6.0 \mathrm{E}-3$ & 36.0 & 9.0 & [48] \\
\hline PDE1p $\rightarrow$ PDE1 & $1.0 \mathrm{E}-1^{\mathrm{a}}$ & & & [48] \\
\hline $\mathrm{PKAC}+\mathrm{PDE} 4 \leftrightarrow \mathrm{PKAc} \_\mathrm{PDE} 4 \rightarrow \mathrm{PKAc}+\mathrm{PDE} 4 \mathrm{p}$ & $6.0 \mathrm{E}-3$ & 36.0 & 9.0 & [48] \\
\hline PDE4p $\rightarrow$ PDE4 & $1.0 \mathrm{E}-1^{\mathrm{a}}$ & & & [48] \\
\hline$\rightarrow \mathrm{Ca}^{2+}$ & $2.5 \mathrm{E}+1^{\mathrm{e}}$ & & & [48] \\
\hline $\mathrm{Ca}^{2+} \rightarrow$ & $0.6^{\mathrm{a}} / 1.7^{\mathrm{a}}$ & & & [48] \\
\hline $2 \mathrm{Ca}^{2+}+$ PP2Bi $\leftrightarrow$ PP2Bi_Ca & $1.0 \mathrm{E}-3^{\mathrm{C}}$ & 1.0 & & [48] \\
\hline $2 \mathrm{Ca}^{2+}+$ PP2Bi_Ca ${ }_{2} \leftrightarrow$ PP2B & $3.0 \mathrm{E}-3^{\mathrm{C}}$ & 1.0 & & [48] \\
\hline $\mathrm{AC5}+\mathrm{Ca}^{2+} \leftrightarrow \mathrm{AC} 5 \_\mathrm{Ca}$ & $1.0 \mathrm{E}-3$ & 0.9 & & [47] \\
\hline$G_{\alpha} G T P+A C 5 \_C a \leftrightarrow G_{\alpha} G T P \_A C 5 \_C a$ & $1.9 \mathrm{E}-2$ & 25.0 & & [47] \\
\hline$G_{\alpha} G T P \_A C 5$ _Ca + ATP $\leftrightarrow G_{\alpha}$ GTP_AC5_Ca_ATP & $6.0 \mathrm{E}-5$ & $1.3 \mathrm{E}-1$ & & [47] \\
\hline$G_{\alpha} G T P \_A C 5$ _Ca_ATP $\leftrightarrow G_{\alpha} G T P \_A C 5$ Ca + CAMP & $14.2^{\mathrm{a}}$ & $1.3 \mathrm{E}-4^{\mathrm{b}}$ & & [47] \\
\hline $\mathrm{PP} 2 \mathrm{~A}+4 \mathrm{Ca}^{2+} \leftrightarrow \mathrm{PP} 2 \mathrm{AC}$ & 7.7E-12 ${ }^{d}$ & $1.0 \mathrm{E}-2$ & & [47] \\
\hline $\mathrm{PP} 2 \mathrm{~A}+\mathrm{PKAC} \leftrightarrow \mathrm{PP} 2 \mathrm{~A} \_\mathrm{PKAc} \rightarrow \mathrm{PP} 2 \mathrm{Ap}+\mathrm{PKAC}$ & $2.5 \mathrm{E}-3$ & 0.3 & 0.1 & [49] \\
\hline PP2Ap $\rightarrow$ PP2A & $4.0 \mathrm{E}-3^{\mathrm{a}}$ & & & [47] \\
\hline $\mathrm{CK} 1 \rightarrow \mathrm{CK} 1 \mathrm{p}$ & $1.0^{\mathrm{a}}$ & & & [47] \\
\hline $\mathrm{PP} 2 \mathrm{~B}+\mathrm{CK} 1 \mathrm{p} \leftrightarrow$ PP2B_CK1p $\rightarrow$ PP2B + CK1 & $3.0 \mathrm{E}-2$ & 24.0 & 6.0 & [47] \\
\hline $\mathrm{CDK} 5+\mathrm{Ca}^{2+} \leftrightarrow \mathrm{CDK} 5 \mathrm{C}$ & $3.0 \mathrm{E}-3$ & 1.0 & & \\
\hline
\end{tabular}

${ }^{a}$ : Unit in $\mathrm{s}^{-1} ;{ }^{b}$ : Unit in $\mathrm{nM}^{-1} \cdot \mathrm{s}^{-1} ;{ }^{\mathrm{c}}$ : Unit in $\mathrm{nM}^{-2} \cdot \mathrm{s}^{-1} ;{ }^{\mathrm{d}}$ : Unit in $\mathrm{nM}^{-4} \cdot \mathrm{s}^{-1} ;{ }^{\text {e }}$ : Unit in $\mathrm{nM} \cdot \mathrm{s}^{-1}$

\#: For a chemical reaction, $\mathrm{K}_{\mathrm{f}}$ is the rate constant for the forward process, $\mathrm{K}_{\mathrm{b}}$ is the rate constant for the backward process, while $\mathrm{K}_{\mathrm{c}}$ is the rate constant for the catalytic step in a Michaelis-Menten kinetics. 
Table 3 Reactions and rate constants of DARPP-32 phosphorylation in dendrites of medium spiny neurons in the striatum (see legend of Figure 1 for abbreviations)

\begin{tabular}{|c|c|c|c|c|}
\hline Reaction & $\mathrm{K}_{\mathrm{f}}\left(\mathrm{nM}^{-1} \cdot \mathrm{s}^{-1}\right)^{\#}$ & $K_{b}\left(s^{-1}\right)$ & $\mathrm{K}_{\mathrm{c}}\left(\mathrm{s}^{-1}\right)$ & Ref. \\
\hline $\mathrm{D}+\mathrm{PKAC} \leftrightarrow \mathrm{D} \_\mathrm{PKAC} \rightarrow \mathrm{D} 34+\mathrm{PKAc}$ & $5.6 \mathrm{E}-3$ & 10.8 & 2.7 & {$[16,50]$} \\
\hline $\mathrm{D} 34+\mathrm{PP} 2 \mathrm{~B} \leftrightarrow \mathrm{D} 34 \_\mathrm{PP} 2 \mathrm{~B} \rightarrow \mathrm{D}+\mathrm{PP} 2 \mathrm{~B}$ & $1.0 \mathrm{E}-3$ & 2.0 & 0.5 & [19] \\
\hline $\mathrm{D}+\mathrm{CDK} 5 \leftrightarrow \mathrm{D} \_\mathrm{CDK} 5 \rightarrow \mathrm{D} 75+\mathrm{CDK} 5$ & $4.5 \mathrm{E}-4$ & 2.0 & 0.5 & {$[47]$} \\
\hline $\mathrm{D} 75+\mathrm{PP} 2 \mathrm{Ap} \leftrightarrow \mathrm{D} 75 \_\mathrm{PP} 2 \mathrm{Ap} \rightarrow \mathrm{D}+\mathrm{PP} 2 \mathrm{Ap}$ & 4.0E-4 & 12.0 & 3.0 & [47] \\
\hline $\mathrm{D} 75+\mathrm{PP} 2 \mathrm{~A} \leftrightarrow \mathrm{D} 75 \_\mathrm{PP} 2 \mathrm{~A} \rightarrow \mathrm{D}+\mathrm{PP} 2 \mathrm{~A}$ & $1.0 \mathrm{E}-4$ & 6.4 & 1.6 & {$[47]$} \\
\hline $\mathrm{D} 75+\mathrm{PP} 2 \mathrm{AC} \leftrightarrow$ D75_PP2AC $\rightarrow \mathrm{D}+\mathrm{PP} 2 \mathrm{Ac}$ & 4.0E-4 & 12.0 & 3.0 & [47] \\
\hline $\mathrm{D}+\mathrm{CK} 2 \leftrightarrow \mathrm{D}_{-} \mathrm{CK} 2 \rightarrow \mathrm{D} 102+\mathrm{CK} 2$ & 4.0E-4 & 6.4 & 1.6 & [51] \\
\hline $\mathrm{D} 102 \rightarrow \mathrm{D}$ & $1.6^{\mathrm{a}}$ & & & \\
\hline $\mathrm{D}+\mathrm{CK} 1 \leftrightarrow \mathrm{D} \_\mathrm{CK} 1 \rightarrow \mathrm{D} 137+\mathrm{CK} 1$ & 4.4E-3 & 12.0 & 3.0 & [25] \\
\hline $\mathrm{D} 137+\mathrm{PP} 2 \mathrm{C} \leftrightarrow \mathrm{D} 137 \_\mathrm{PP} 2 \mathrm{C} \rightarrow \mathrm{D}+\mathrm{PP} 2 \mathrm{C}$ & $7.5 \mathrm{E}-3$ & 12.0 & 3.0 & [47] \\
\hline $\mathrm{D} 102+\mathrm{PKAC} \leftrightarrow \mathrm{D} 102 \_\mathrm{PKAC} \rightarrow \mathrm{D} 34: 102+\mathrm{PKAC}$ & $5.6 \mathrm{E}-3$ & 10.8 & 2.7 & \\
\hline D34:102 + PP2B $\leftrightarrow$ D34:102_PP2B $\rightarrow$ D102 + PP2B & $7.5 \mathrm{E}-5$ & 0.12 & 0.03 & \\
\hline D137 + PKAC $\leftrightarrow$ D137_PKAc $\rightarrow$ D34:137 + PKAC & $5.6 \mathrm{E}-3$ & 10.8 & 2.7 & {$[16,50]$} \\
\hline $\mathrm{D} 34: 137+\mathrm{PP} 2 \mathrm{~B} \leftrightarrow \mathrm{D} 34: 137 \_\mathrm{PP} 2 \mathrm{~B} \rightarrow \mathrm{D} 137+\mathrm{PP} 2 \mathrm{~B}$ & $7.5 \mathrm{E}-5$ & 0.12 & 0.03 & [25] \\
\hline D102 + CDK5 ↔ D102_CDK5 $\rightarrow$ D75:102 + CDK5 & $4.5 \mathrm{E}-4$ & 2.0 & 0.5 & \\
\hline $\mathrm{D} 75: 102+\mathrm{PP} 2 \mathrm{Ap} \leftrightarrow \mathrm{D} 75: 102 \_P P 2 A p \rightarrow \mathrm{D} 102+\mathrm{PP} 2 \mathrm{Ap}$ & 4.0E-4 & 12.0 & 3.0 & \\
\hline $\mathrm{D} 75: 102+\mathrm{PP} 2 \mathrm{~A} \leftrightarrow \mathrm{D} 75: 102 \_\mathrm{PP} 2 \mathrm{~A} \rightarrow \mathrm{D} 102+\mathrm{PP} 2 \mathrm{~A}$ & $1.0 \mathrm{E}-4$ & 6.4 & 1.6 & \\
\hline $\mathrm{D} 75: 102+\mathrm{PP} 2 \mathrm{AC} \leftrightarrow \mathrm{D} 75: 102 \_\mathrm{PP} 2 \mathrm{AC} \rightarrow \mathrm{D} 102+\mathrm{PP} 2 \mathrm{AC}$ & 4.0E-4 & 12.0 & 3.0 & \\
\hline $\mathrm{D} 137+\mathrm{CDK5} \leftrightarrow \mathrm{D} 137 \_\mathrm{CDK} 5 \rightarrow \mathrm{D} 75: 137+\mathrm{CDK5}$ & 4.5E-4 & 2.0 & 0.5 & \\
\hline D75:137 + PP2Ap $\leftrightarrow$ D75:137_PP2Ap $\rightarrow$ D137 + PP2Ap & 4.0E-4 & 12.0 & 3.0 & \\
\hline D75:137 + PP2A $\leftrightarrow$ D75:137_PP2A $\rightarrow$ D137 + PP2A & $1.0 \mathrm{E}-4$ & 6.4 & 1.6 & \\
\hline $\mathrm{D} 75: 137+\mathrm{PP} 2 \mathrm{AC} \leftrightarrow \mathrm{D} 75: 137 \_\mathrm{PP} 2 \mathrm{Ac} \rightarrow \mathrm{D} 137+\mathrm{PP} 2 \mathrm{Ac}$ & 4.0E-4 & 12.0 & 3.0 & \\
\hline $\mathrm{D} 34+\mathrm{CK} 2 \leftrightarrow \mathrm{D} 34 \_\mathrm{CK} 2 \rightarrow \mathrm{D} 34: 102+\mathrm{CK} 2$ & 4.0E-4 & 6.4 & 1.6 & \\
\hline D34:102 $\rightarrow$ D34 & $1.6^{\mathrm{a}}$ & & & \\
\hline $\mathrm{D} 75+\mathrm{CK} 2 \leftrightarrow \mathrm{D} 75 \_\mathrm{CK} 2 \rightarrow \mathrm{D} 75: 102+\mathrm{CK} 2$ & 4.0E-4 & 6.4 & 1.6 & \\
\hline $\mathrm{D} 75: 102 \rightarrow \mathrm{D} 75$ & $1.6^{\mathrm{a}}$ & & & \\
\hline $\mathrm{D} 34+\mathrm{CK} 1 \leftrightarrow \mathrm{D} 34 \_\mathrm{CK} 1 \rightarrow \mathrm{D} 34: 137+\mathrm{CK} 1$ & 4.4E-3 & 12.0 & 3.0 & [25] \\
\hline D34:137 + PP2C $\leftrightarrow$ D34:137_PP2C $\rightarrow$ D34 + PP2C & $7.5 \mathrm{E}-3$ & 12.0 & 3.0 & [47] \\
\hline $\mathrm{D} 75+\mathrm{CK} 1 \leftrightarrow \mathrm{D} 75 \_\mathrm{CK} 1 \rightarrow \mathrm{D} 75: 137+\mathrm{CK} 1$ & 4.4E-3 & 12.0 & 3.0 & [25] \\
\hline $\mathrm{D} 75: 137+\mathrm{PP2C} \leftrightarrow \mathrm{D} 75: 137 \_\mathrm{PP} 2 \mathrm{C} \rightarrow \mathrm{D} 75+\mathrm{PP} 2 \mathrm{C}$ & $7.5 \mathrm{E}-3$ & 12.0 & 3.0 & [47] \\
\hline $\mathrm{D} 102+\mathrm{CK} 1 \leftrightarrow \mathrm{D} 102 \_\mathrm{CK} 1 \rightarrow \mathrm{D} 102: 137+\mathrm{CK} 1$ & $4.4 \mathrm{E}-3$ & 12.0 & 3.0 & \\
\hline $\mathrm{D} 102: 137+\mathrm{PP} 2 \mathrm{C} \leftrightarrow \mathrm{D} 102: 137 \_\mathrm{PP} 2 \mathrm{C} \rightarrow \mathrm{D} 102+\mathrm{PP} 2 \mathrm{C}$ & $7.5 \mathrm{E}-3$ & 12.0 & 3.0 & \\
\hline $\mathrm{D} 137+\mathrm{CK} 2 \leftrightarrow \mathrm{D} 137 \_\mathrm{CK} 2 \rightarrow \mathrm{D} 102: 137+\mathrm{CK} 2$ & 4.0E-4 & 6.4 & 1.6 & \\
\hline $\mathrm{D} 102: 137 \rightarrow \mathrm{D} 137$ & $1.6^{\mathrm{a}}$ & & & \\
\hline D34:102 + CK1 ↔ D34:102_CK1 $\rightarrow$ D34:102:137 + CK1 & $4.4 \mathrm{E}-3$ & 12.0 & 3.0 & \\
\hline D34:102:137 + PP2C ↔ D34:102:137_PP2C $\rightarrow$ D34:102 + PP2C & $7.5 \mathrm{E}-3$ & 12.0 & 3.0 & \\
\hline D34:137 + CK2 ↔ D34:137_CK2 $\rightarrow$ D34:102:137 + CK2 & 4.0E-4 & 6.4 & 1.6 & \\
\hline D34:102:137 $\rightarrow$ D34:137 & $1.6^{\mathrm{a}}$ & & & \\
\hline D102:137 + PKAc $\leftrightarrow$ D102:137_PKAc $\rightarrow$ D34:102:137 + PKAc & $5.6 \mathrm{E}-3$ & 10.8 & 2.7 & \\
\hline D34:102:137 + PP2B $\leftrightarrow$ D34:102:137_PP2B $\rightarrow$ D102:137 + PP2B & $7.5 \mathrm{E}-5$ & 0.12 & 0.03 & \\
\hline $\mathrm{D} 75: 102+\mathrm{CK} 1 \leftrightarrow \mathrm{D} 75: 102 \_\mathrm{CK} 1 \rightarrow \mathrm{D} 75: 102: 137+\mathrm{CK} 1$ & 4.4E-3 & 12.0 & 3.0 & \\
\hline D75:102:137 + PP2C $\leftrightarrow$ D75:102:137_PP2C $\rightarrow$ D75:102 + PP2C & $7.5 \mathrm{E}-3$ & 12.0 & 3.0 & \\
\hline $\mathrm{D} 75: 137+\mathrm{CK} 2 \leftrightarrow \mathrm{D} 75: 137 \_C K 2 \rightarrow \mathrm{D} 75: 102: 137+\mathrm{CK} 2$ & 4.0E-4 & 6.4 & 1.6 & \\
\hline $\mathrm{D} 75: 102: 137 \rightarrow \mathrm{D} 75: 137$ & $1.6^{\mathrm{a}}$ & & & \\
\hline D102:137 + CDK5 ↔ D102:137_CDK5 $\rightarrow$ D75:102:137 + CDK5 & 4.5E-4 & 2.0 & 0.5 & \\
\hline D75:102:137 + PP2Ap $\leftrightarrow$ D75:102:137_PP2Ap $\rightarrow$ D102:137 + PP2Ap & 4.0E-4 & 12.0 & 3.0 & \\
\hline D75:102:137 + PP2A $\leftrightarrow$ D75:102:137_PP2A $\rightarrow$ D102:137 + PP2A & $1.0 \mathrm{E}-4$ & 6.4 & 1.6 & \\
\hline $\mathrm{D} 75: 102: 137+\mathrm{PP} 2 \mathrm{Ac} \leftrightarrow \mathrm{D} 75: 102: 137 \_P P 2 A C \rightarrow \mathrm{D} 102: 137+\mathrm{PP} 2 \mathrm{Ac}$ & 4.0E-4 & 12.0 & 3.0 & \\
\hline D34 + PP1 ↔ D34_PP1 & 0.4 & 0.6 & & [47] \\
\hline $\mathrm{D} 34 \_\mathrm{PP} 1+\mathrm{PP} 2 \mathrm{~B} \leftrightarrow \mathrm{D} 34$ PP1_PP2B $\rightarrow \mathrm{D}+\mathrm{PP} 1+\mathrm{PP} 2 \mathrm{~B}$ & $1.0 \mathrm{E}-3$ & 2.0 & 0.5 & [47] \\
\hline
\end{tabular}


Table 3: Reactions and rate constants of DARPP-32 phosphorylation in dendrites of medium spiny neurons in the striatum (see legend of Figure 1 for abbreviations) (Continued)

\begin{tabular}{|c|c|c|c|c|}
\hline D34:102 + PP1 ↔ D34:102_PP1 & 0.4 & 0.6 & & \\
\hline D34:102_PP1 + PP2B $\leftrightarrow$ D34:102_PP1_PP2B $\rightarrow$ D102 + PP1 + PP2B & $1.0 \mathrm{E}-3$ & 2.0 & 0.5 & \\
\hline D34:137 + PP1 ↔ D34:137_PP1 & 0.4 & 0.6 & & \\
\hline D34:137_PP1 + PP2B $\leftrightarrow$ D34:137_PP1_PP2B $\rightarrow$ D137 + PP1 + PP2B & $1.0 \mathrm{E}-3$ & 2.0 & 0.5 & \\
\hline D34:102:137 + PP1 ↔ D34:102:137_PP1 & 0.4 & 0.6 & & \\
\hline D34:102:137_PP1 + PP2B ↔ D34:102:137_PP1_PP2B $\rightarrow$ D102:137 + PP1 + PP2B & $1.0 \mathrm{E}-3$ & 2.0 & 0.5 & \\
\hline D75 + PKAC $\leftrightarrow$ D75_PKAC & $5.6 \mathrm{E}-3$ & 10.8 & & [16] \\
\hline D75:102 + PKAC $\leftrightarrow$ D75:102_PKAC & $5.6 \mathrm{E}-3$ & 10.8 & & \\
\hline D75:137 + PKAC $\leftrightarrow$ D75:137_PKAC & $5.6 \mathrm{E}-3$ & 10.8 & & \\
\hline D75:102:137 + PKAc ↔ D75:102:137_PKAc & $5.6 \mathrm{E}-3$ & 10.8 & & \\
\hline
\end{tabular}

a: Unit in $\mathrm{s}^{-1}$

\#: For a chemical reaction, $\mathrm{K}_{\mathrm{f}}$ is the rate constant for the forward process, $\mathrm{K}_{\mathrm{b}}$ is the rate constant for the backward process, while $\mathrm{K}_{\mathrm{c}}$ is the rate constant for the catalytic step in a Michaelis-Menten kinetics.

Table 4 Initial values for the DARPP-32 phosphorylation system in dendrites of medium spiny neurons in the striatum (see legend of Figure 1 for abbreviations)

\begin{tabular}{ccc}
\hline Molecule & Concentration (nM) & Reference \\
\hline DA & 10.0 & {$[45]$} \\
D1 & $5.0 \mathrm{E}+2$ & {$[45]$} \\
$G_{\alpha \beta \gamma}$ & $3.0 \mathrm{E}+3$ & {$[45]$} \\
AC5 & $2.5 \mathrm{E}+3$ & {$[45]$} \\
ATP & $2.0 \mathrm{E}+6$ & {$[45]$} \\
PDE1 & $4.0 \mathrm{E}+3$ & {$[45]$} \\
PDE4 & $2.0 \mathrm{E}+3$ & {$[45]$} \\
DARPP-32 & $5.0 \mathrm{E}+4$ & {$[52]$} \\
PKA & $1.2 \mathrm{E}+3$ & {$[53]$} \\
PP2Bi & $4.0 \mathrm{E}+3$ & {$[45]$} \\
CDK5 & $1.8 \mathrm{E}+3$ & {$[45]$} \\
PP2A & $2.0 \mathrm{E}+3$ & {$[54,55]$} \\
CK1 & $2.0 \mathrm{E}+3$ & \\
PP2C & $2.0 \mathrm{E}+3$ & {$[56,57]$} \\
PP1 & $5.0 \mathrm{E}+3$ & \\
CK2 & $2.0 \mathrm{E}+3$ &
\end{tabular}

Figure 1 indicates that dopamine can bind to its D1 receptors on the postsynaptic membrane, where it forms a complex with the corresponding $G$ protein. The complex releases its alpha subunit, which binds to adenylate cyclase (AC5) and generates cAMP. This second messenger binds to the regulatory subunit of PKA and releases its catalytic subunit, which phosphorylates DARPP-32 at threonine 34 (Thr34, positioned according to the rat sequence). Counteracting this effect, the same site is dephosphorylated by the phosphatase PP2B. DARPP-32 can also be phosphorylated at a second threonine site ( Thr75, positioned according to the rat sequence) by CDK5, a process which is counteracted by protein phosphatase $2 \mathrm{~A}$ (PP2A), which mediates the release of the phosphate group from this site. DARPP-32 possesses two further phosphorylation sites, namely serine 102 and serine 137 (Ser102 and Ser137, positioned according to the rat sequence). Casein kinase 1 (CK1) and casein kinase 2 (CK2) execute the phosphorylation at Ser137 and Ser102, respectively.

These phosphorylation sites of DARPP-32 can be phosphorylated simultaneously, and transitions between different phosphorylation configurations are illustrated in Figure 2. However, phosphorylation activities at these sites are not independent of each other. For example, Ser102 and Ser137 phosphorylation of DARPP-32 can activate phosphorylation and repress dephosphorylation of DARPP-32 at Thr34, respectively. Importantly, DARPP-32 with Thr75 phosphorylation can bind to PKA and inhibit its capability to phosphorylate DARPP32 at Thr34, which permits us to exclude the combined phosphorylation form of Thr34-Thr75 [16,47]. It is evident that the signal cascade becomes a complicated network when all possible interactions are taken into account.

We model this signaling system with ordinary differential equations for all variables (molecules) in Tables 2 and 3 according to the law of mass action. Each process is formulated as a product of concentrations of contributors raised to the relevant reaction orders (most of which are 1), and multiplied with the appropriate rate constant. An enzymatic reaction is assumed to follow a reversible Michaelis-Menten mechanism with forward, backward, and catalytic steps that are quantified by the rate constants $K_{f}, K_{b}$, and $K_{c}$, respectively. A non-enzymatic reaction is treated with both forward and backward steps or as a single irreversible forward step reaction. The derivative of each variable is determined by the difference between the sum of its influxes and the sum of its effluxes; the equations are explicitly given in Additional file 1. The dynamics of the signaling network is obtained as the solution of the system of equations. 
To gain quantitative insights, values for rate constants, reaction orders, and initial variable states are needed. This information is presented in Tables 2, 3 and 4 , which were populated with information from the literature and databases such as BRENDA http:// www.brenda-enzymes.info/. Some values were computed from kinetic data, while others (mostly rate constants for transitions between different forms of phosphorylation) were specified with reasonable estimates.

Once the numerical characterization was completed, it was straightforward to test different scenarios, for instance, by exposing the system to various signals and to random perturbations. Representative input patterns consist of combinations of the following signal specifications:

a. individual or combined dopamine and glutamate signals;

b. transient or sustained signals;

c. one-shot pulse or repetitive pulse signals;

d. signals of varying length;

e. signals of high or low amplitude.

The input signals were simulated in terms of different combinations of these specifications, and their effects on signal integration by DARPP-32 without or with perturbations were systematically evaluated.

The responses to the different input signals were examined from two distinct and relevant viewpoints. First, long-term effects indicate whether the system can switch to a different steady state, and if so, how large in magnitude the change is. Second, the transient effects, which are obtained as the dynamic behavior, reflect short-term responses of the system. Critical output variables for these responses are PKA and PP1. In addition to these two indicators, the phosphorylation profile of DARPP-32 can serve as a valuable sentinel, because it represents the internal state of the signal transduction system, while PKA and PP1 are functional proteins performing biochemical, cellular, and physiological regulations.

Additional file 1: The DARPP-32 model. This is a computational model of the DARPP-32 system in the human striatum using ordinary differential equations.

Click here for file

[http://www.biomedcentral.com/content/supplementary/1752-0509-4-26S1.DOC]

\section{Acknowledgements}

This work was supported by a grant from the National Institutes of Health (P01-ES016731, G.W.M. PI) and an endowment from the Georgia Research Alliance (E.O.V). Any opinions, findings, and conclusions or recommendations expressed in this material are those of the authors and do not necessarily reflect the views of the sponsoring institutions.

\section{Author details}

${ }^{1}$ Department of Biomedical Engineering, Georgia Institute of Technology and Emory University Medical School, Atlanta, GA 30332, USA. ${ }^{2}$ Center for Neurodegenerative Disease, Emory University School of Medicine, Atlanta, GA 30322, USA. ${ }^{3}$ Integrative BioSystems Institute, Georgia Institute of Technology, Atlanta, GA 30332, USA. ${ }^{4}$ Department of Environmental and Occupational Health, Rollins School of Public Health, Emory University, Atlanta, GA 30322, USA.

\section{Authors' contributions}

ZQ carried out the modeling, analyses and interpretation of results, and manuscript writing. GWM participated in the design of the work and help to draft the manuscript. EOV conceived the study, interpreted results, and wrote the manuscript. All authors have read and approved the manuscript.

Received: 3 December 2009 Accepted: 17 March 2010

Published: 17 March 2010

\section{References}

1. Graybiel AM: Neurotransmitters and neuromodulators in the basal ganglia. Trends Neurosci 1990, 13:244-254

2. Walaas SI, Aswad DW, Greengard P: A dopamine- and cyclic AMPregulated phosphoprotein enriched in dopamine-innervated brain regions. Nature 1983, 301:69-71.

3. Svenningsson P, Nishi A, Fisone G, Girault JA, Nairn AC, Greengard P: DARPP-32: an integrator of neurotransmission. Annu Rev Pharmacol Toxicol 2004, 44:269-296.

4. Greengard P, Allen PB, Nairn AC: Beyond the dopamine receptor: the DARPP-32/protein phosphatase-1 cascade. Neuron 1999, 23:435-447.

5. Fienberg AA, Hiroi N, Mermelstein PG, Song W, Snyder GL, Nishi A, Cheramy A, O'Callaghan JP, Miller DB, Cole DG, et al: DARPP-32: regulator of the efficacy of dopaminergic neurotransmission. Science 1998, 281:838-842.

6. Fienberg AA, Greengard P: The DARPP-32 knockout mouse. Brain Res Brain Res Rev 2000, 31:313-319.

7. Spencer HJ: Antagonism of cortical excitation of striatal neurons by glutamic acid diethyl ester: evidence for glutamic acid as an excitatory transmitter in the rat striatum. Brain Res 1976, 102:91-101.

8. Girault JA, Spampinato U, Savaki HE, Glowinski J, Besson MJ: In vivo release of $[3 \mathrm{H}]$ gamma-aminobutyric acid in the rat neostriatum-I. Characterization and topographical heterogeneity of the effects of dopaminergic and cholinergic agents. Neuroscience 1986, 19:1101-1108.

9. Nishi A, Kuroiwa M, Miller DB, O'Callaghan JP, Bateup HS, Shuto T, Sotogaku N, Fukuda T, Heintz N, Greengard P, Snyder GL: Distinct roles of PDE4 and PDE10A in the regulation of CAMP/PKA signaling in the striatum. J Neurosci 2008, 28:10460-10471.

10. Hemmings HC Jr, Greengard P, Tung HY, Cohen P: DARPP-32, a dopamine-regulated neuronal phosphoprotein, is a potent inhibitor of protein phosphatase-1. Nature 1984, 310:503-505.

11. Kesavapany S, Li BS, Amin N, Zheng YL, Grant P, Pant HC: Neuronal cyclindependent kinase 5 : role in nervous system function and its specific inhibition by the Cdk5 inhibitory peptide. Biochim Biophys Acta 2004, 1697:143-153.

12. Kerokoski $P$, Suuronen $T$, Salminen A, Soininen H, Pirttila T: Cleavage of the cyclin-dependent kinase 5 activator p35 to p25 does not induce tau hyperphosphorylation. Biochem Biophys Res Commun 2002, 298:693-698.

13. Dhavan $\mathrm{R}$, Greer PL, Morabito MA, Orlando LR, Tsai LH: The cyclindependent kinase 5 activators p35 and p39 interact with the alphasubunit of $\mathrm{Ca} 2+/$ calmodulin-dependent protein kinase II and alphaactinin-1 in a calcium-dependent manner. J Neurosci 2002, 22:7879-7891.

14. Nishi A, Snyder GL, Greengard P: Bidirectional regulation of DARPP-32 phosphorylation by dopamine. J Neurosci 1997, 17:8147-8155.

15. Jin M, Bateup H, Padovan JC, Greengard P, Nairn AC, Chait BT: Quantitative analysis of protein phosphorylation in mouse brain by hypothesis-driven multistage mass spectrometry. Anal Chem 2005, 77:7845-7851.

16. Bibb JA, Snyder GL, Nishi A, Yan Z, Meijer L, Fienberg AA, Tsai LH, Kwon YT, Girault JA, Czernik AJ, et al: Phosphorylation of DARPP-32 by Cdk5 modulates dopamine signalling in neurons. Nature 1999, 402:669-671. 
17. Snyder GL, Galdi S, Fienberg AA, Allen P, Nairn AC, Greengard P: Regulation of AMPA receptor dephosphorylation by glutamate receptor agonists. Neuropharmacology 2003, 45:703-713.

18. Walaas SI, Greengard P: DARPP-32, a dopamine- and adenosine 3':5'monophosphate-regulated phosphoprotein enriched in dopamineinnervated brain regions. I. Regional and cellular distribution in the rat brain. J Neurosci 1984, 4:84-98.

19. Nishi A, Bibb JA, Snyder GL, Higashi H, Nairn AC, Greengard P. Amplification of dopaminergic signaling by a positive feedback loop. Proc Natl Acad Sci USA 2000, 97:12840-12845.

20. Brown AM, Deutch AY, Colbran RJ: Dopamine depletion alters phosphorylation of striatal proteins in a model of Parkinsonism. Eur $J$ Neurosci 2005, 22:247-256.

21. Fischer W, Franke H, Scheibler P, Allgaier C, Illes P: AMPA-induced Ca(2+) influx in cultured rat cortical nonpyramidal neurones: pharmacological characterization using fura-2 microfluorimetry. Eur J Pharmacol 2002 , 438:53-62

22. Nishi A, Bibb JA, Matsuyama S, Hamada M, Higashi H, Nairn AC, Greengard P: Regulation of DARPP-32 dephosphorylation at PKA- and Cdk5-sites by NMDA and AMPA receptors: distinct roles of calcineurin and protein phosphatase-2A. J Neurochem 2002, 81:832-841.

23. Chergui $K$, Svenningsson P, Greengard P: Cyclin-dependent kinase 5 regulates dopaminergic and glutamatergic transmission in the striatum. Proc Natl Acad Sci USA 2004, 101:2191-2196.

24. Chen PC, Chen JC: Enhanced Cdk5 activity and p35 translocation in the ventral striatum of acute and chronic methamphetamine-treated rats. Neuropsychopharmacology 2005, 30:538-549.

25. Takahashi S, Ohshima T, Cho A, Sreenath T, ladarola MJ, Pant HC, Kim Y, Nairn AC, Brady RO, Greengard P, Kulkarni AB: Increased activity of cyclindependent kinase 5 leads to attenuation of cocaine-mediated dopamine signaling. Proc Natl Acad Sci USA 2005, 102:1737-1742.

26. Nishi $A$, Watanabe $Y$, Higashi $H$, Tanaka M, Nairn AC, Greengard P: Glutamate regulation of DARPP-32 phosphorylation in neostriatal neurons involves activation of multiple signaling cascades. Proc Natl Acad Sci USA 2005, 102:1199-1204

27. Sun JY, Wu XS, Wu LG: Single and multiple vesicle fusion induce different rates of endocytosis at a central synapse. Nature 2002, 417:555-559.

28. Meinrenken CJ, Borst JG, Sakmann B: Local routes revisited: the space and time dependence of the $\mathrm{Ca} 2+$ signal for phasic transmitter release at the rat calyx of Held. J Physiol 2003, 547:665-689.

29. Fisone G, Hakansson K, Borgkvist A, Santini E: Signaling in the basal ganglia: postsynaptic and presynaptic mechanisms. Physiol Behav 2007, 92:8-14.

30. Greengard P: The neurobiology of slow synaptic transmission. Science 2001, 294:1024-1030.

31. Yan Z, Hsieh-Wilson L, Feng J, Tomizawa K, Allen PB, Fienberg AA, Nairn AC, Greengard P: Protein phosphatase 1 modulation of neostriatal AMPA channels: regulation by DARPP-32 and spinophilin. Nat Neurosci 1999, 2:13-17.

32. Flores-Hernandez J, Hernandez S, Snyder GL, Yan Z, Fienberg AA, Moss SJ, Greengard $P$, Surmeier $D J: D(1)$ dopamine receptor activation reduces GABA(A) receptor currents in neostriatal neurons through a PKA/DARPP32/PP1 signaling cascade. J Neurophysiol 2000, 83:2996-3004.

33. Flores-Hernandez J, Cepeda C, Hernandez-Echeagaray E, Calvert CR, Jokel ES, Fienberg AA, Greengard P, Levine MS: Dopamine enhancement of NMDA currents in dissociated medium-sized striatal neurons: role of D1 receptors and DARPP-32. J Neurophysiol 2002, 88:3010-3020.

34. Calabresi P, Gubellini P, Centonze D, Picconi B, Bernardi G, Chergui K, Svenningsson P, Fienberg AA, Greengard P: Dopamine and CAMPregulated phosphoprotein $32 \mathrm{kDa}$ controls both striatal long-term depression and long-term potentiation, opposing forms of synaptic plasticity. J Neurosci 2000, 20:8443-8451.

35. Hyman SE, Malenka RC: Addiction and the brain: the neurobiology of compulsion and its persistence. Nat Rev Neurosci 2001, 2:695-703.

36. Liu FC, Graybiel AM: Spatiotemporal dynamics of CREB phosphorylation: transient versus sustained phosphorylation in the developing striatum. Neuron 1996, 17:1133-1144.

37. Svenningsson $P$, Fienberg $A A$, Allen $P B$, Moine $C L$, Lindskog $M$, Fisone $G$ Greengard $P$, Fredholm BB: Dopamine $D(1)$ receptor-induced gene transcription is modulated by DARPP-32. J Neurochem 2000, 75:248-257.
38. Lee HK, Barbarosie M, Kameyama K, Bear MF, Huganir RL: Regulation of distinct AMPA receptor phosphorylation sites during bidirectional synaptic plasticity. Nature 2000, 405:955-959.

39. Mangiavacchi S, Wolf ME: Stimulation of N-methyl-D-aspartate receptors, AMPA receptors or metabotropic glutamate receptors leads to rapid internalization of AMPA receptors in cultured nucleus accumbens neurons. Eur J Neurosci 2004, 20:649-657.

40. Qi Z, Miller GW, Voit EO: Computational systems analysis of dopamine metabolism. PLOS ONE 2008, 3:e2444.

41. Picconi B, Centonze D, Hakansson K, Bernardi G, Greengard P, Fisone G, Cenci MA, Calabresi P: Loss of bidirectional striatal synaptic plasticity in LDOPA-induced dyskinesia. Nat Neurosci 2003, 6:501-506.

42. Langmead CJ, Watson J, Reavill C: Muscarinic acetylcholine receptors as CNS drug targets. Pharmacol Ther 2008, 117:232-243.

43. Sato K, Ueda H, Okumura F, Misu Y: Supersensitization of intrastriatal dopamine receptors involved in opposite regulation of acetylcholine release in Parkinson's model rats. Neurosci Lett 1994, 173:59-62.

44. Phillips JM, Lam HA, Ackerson LC, Maidment NT: Blockade of mGluR glutamate receptors in the subthalamic nucleus ameliorates motor asymmetry in an animal model of Parkinson's disease. Eur J Neurosci 2006, 23:151-160.

45. Lindskog M, Kim M, Wikstrom MA, Blackwell KT, Kotaleski JH: Transient calcium and dopamine increase PKA activity and DARPP-32 phosphorylation. PLoS Comput Biol 2006, 2:e119.

46. Barbano PE, Spivak M, Flajolet M, Nairn AC, Greengard P, Greengard L: A mathematical tool for exploring the dynamics of biological networks. Proc Natl Acad Sci USA 2007, 104:19169-19174.

47. Fernandez É, Schiappa R, Girault JA, Le Novère N: DARPP-32 is a robust integrator of dopamine and glutamate signals. PLoS Comput Biol 2006, 2 : e176.

48. Bhalla US, lyengar R: Emergent properties of networks of biological signaling pathways. Science 1999, 283:381-387.

49. Usui $H$, Inoue $R$, Tanabe $O$, Nishito $Y$, Shimizu M, Hayashi H, Kagamiyama $H$, Takeda M: Activation of protein phosphatase $2 \mathrm{~A}$ by CAMP-dependent protein kinase-catalyzed phosphorylation of the 74-kDa B" (delta) regulatory subunit in vitro and identification of the phosphorylation sites. FEBS Lett 1998, 430:312-316.

50. Hemmings HC Jr, Nairn AC, Greengard P: DARPP-32, a dopamine- and adenosine $3^{\prime}: 5^{\prime}$-monophosphate-regulated neuronal phosphoprotein. II. Comparison of the kinetics of phosphorylation of DARPP-32 and phosphatase inhibitor 1. J Biol Chem 1984, 259:14491-14497.

51. Girault JA, Hemmings HC Jr, Williams KR, Nairn AC, Greengard P: Phosphorylation of DARPP-32, a dopamine- and CAMP-regulated phosphoprotein, by casein kinase II. J Biol Chem 1989, 264:21748-21759.

52. Hemmings HC Jr, Greengard P: DARPP-32, a dopamine- and adenosine 3':5'-monophosphate-regulated phosphoprotein: regional, tissue, and phylogenetic distribution. J Neurosci 1986, 6:1469-1481.

53. Hofmann F, Bechtel PJ, Krebs EG: Concentrations of cyclic AMPdependent protein kinase subunits in various tissues. J Biol Chem 1977, 252:1441-1447.

54. Nagase $T$, Murakami $T$, Nozaki $H$, Inoue $R$, Nishito $Y$, Tanabe $O$, Usui $H$, Takeda M: Tissue and subcellular distributions, and characterization of rat brain protein phosphatase $2 \mathrm{~A}$ containing a $72-\mathrm{kDa}$ delta/B" subunit. $J$ Biochem 1997, 122:178-187.

55. Sim AT, Ratcliffe E, Mumby MC, Villa-Moruzzi E, Rostas JA: Differential activities of protein phosphatase types 1 and $2 \mathrm{~A}$ in cytosolic and particulate fractions from rat forebrain. J Neurochem 1994, 62:1552-1559.

56. Ouimet CC, da Cruz e Silva EF, Greengard P: The alpha and gamma 1 isoforms of protein phosphatase 1 are highly and specifically concentrated in dendritic spines. Proc Natl Acad Sci USA 1995, 92:3396-3400.

57. Desdouits F, Cheetham JJ, Huang HB, Kwon YG, da Cruz e Silva EF, Denefle P, Ehrlich ME, Nairn AC, Greengard P, Girault JA: Mechanism of inhibition of protein phosphatase 1 by DARPP-32: studies with recombinant DARPP-32 and synthetic peptides. Biochem Biophys Res Commun 1995, 206:652-658.

doi:10.1186/1752-0509-4-26

Cite this article as: Qi et al: The internal state of medium spiny neurons varies in response to different input signals. BMC Systems Biology 2010 4:26 